\title{
High field fMRI reveals thalamocortical integration of segregated cognitive and emotional processing in mediodorsal and intralaminar thalamic nuclei
}

\section{D. Metzger ${ }^{1}$, U. Eckert', J. Steiner ${ }^{1}$, A. Sartorius ${ }^{2}$, J. E. Buchmann ${ }^{1}$, J. Stadler ${ }^{3}$, C. Tempelmann ${ }^{4}$, O. Speck ${ }^{5}$, B. Bogerts ${ }^{1}$, B. Abler ${ }^{6}$ and M. Walter ${ }^{1 *}$}

\author{
Department of Psychiatry, Otto-von-Guericke University, Magdeburg, Germany \\ 2 Department of Psychiatry and Psychotherapy, Central Institute for Mental Health, Mannheim, Germany \\ 3 Leibniz Institute for Neurobiology, Magdeburg, Germany \\ 4 Department of Neurology, Otto-von-Guericke University, Magdeburg, Germany \\ 5 Biomedical Magnetic Resonance, Otto-von-Guericke University, Magdeburg, Germany \\ ${ }^{6}$ Department of Psychiatry, University of UIm, UIm, Germany
}

\section{Edited by:}

Jose L. Lanciego, University of

Navarra, Spain

\section{Reviewed by:}

Christian Windischberger, Medizinische

Universität Wien, Austria

Simone Grimm, Psychiatric University

Hospital Zurich, Switzerland

${ }^{*}$ Correspondence:

M. Walter, Clinical Affective

Neuroimaging Laboratory, Department

of Psychiatry, Otto-von-Guericke

University Magdeburg, Leipziger

Strasse 44, 39120 Magdeburg,

Germany.

e-mail:martin@canlab.de
Thalamocortical loops, connecting functionally segregated, higher order cortical regions, and basal ganglia, have been proposed not only for well described motor and sensory regions, but also for limbic and prefrontal areas relevant for affective and cognitive processes. These functions are, however, more specific to humans, rendering most invasive neuroanatomical approaches impossible and interspecies translations difficult. In contrast, non-invasive imaging of functional neuroanatomy using $\mathrm{fMRI}$ allows for the development of elaborate task paradigms capable of testing the specific functionalities proposed for these circuits. Until recently, spatial resolution largely limited the anatomical definition of functional clusters at the level of distinct thalamic nuclei. Since their anatomical distinction seems crucial not only for the segregation of cognitive and limbic loops but also for the detection of their functional interaction during cognitive-emotional integration, we applied high resolution fMRI on 7 Tesla. Using an eventrelated design, we could isolate thalamic effects for preceding attention as well as experience of erotic stimuli. We could demonstrate specific thalamic effects of general emotional arousal in mediodorsal nucleus and effects specific to preceding attention and expectancy in intralaminar centromedian/parafascicular complex. These thalamic effects were paralleled by specific coactivations in the head of caudate nucleus as well as segregated portions of rostral or caudal cingulate cortex and anterior insula supporting distinct thalamo-striato-cortical loops. In addition to predescribed effects of sexual arousal in hypothalamus and ventral striatum, high resolution $\mathrm{fMRI}$ could extent this network to paraventricular thalamus encompassing laterodorsal and parataenial nuclei. We could lend evidence to segregated subcortical loops which integrate cognitive and emotional aspects of basic human behavior such as sexual processing.

Keywords: salience processing, centromedian/parafascicular thalamus, mediodorsal thalamus, basal ganglia, cognition, emotion, high field fMRI, sexual processing

\section{INTRODUCTION}

Functional imaging studies of the last decade have vastly illustrated the importance of cortical networks for the highly differentiated subfunctions of human behavior, while the role of subcortical structures, foremost the basal ganglia, in guiding human behavior has not been investigated to an equal extent. This is mainly due to the fact that these comparably small structures remained difficult to characterize given the limited spatial resolutions of noninvasive imaging techniques such as fMRI. However, a huge body of literature from animal studies or clinical insights from brain lesions exists, which suggests that the elaborate set of cortical functional networks may be orchestrated by anatomically well-defined subcortical structures. Since subcortical components of corticosubcortical networks could not be sufficiently characterized so far, their functional segregation based on non-invasive imaging studies seems crucial to understanding the brain's functional architecture as a whole. Existing concepts, as suggested by Alexander and others, rely on segregated but integrating circuits, some of them forming functional subsystems or cortico-striato-thalamic "loops" (Alexander et al., 1986).

These integrated loops may also control basic processes such as sexual arousal which has been previously characterized as multidimensional, spanning various functional systems and brain areas in humans. During processing of sexually salient information, cognitive, motivational, emotional, and autonomic processes can be discerned which contribute to different aspects of the subjective experience and which are known to interact heavily (Redouté et al., 2000). Recent studies could differentiate subsystems involved in processing of either specific sexual arousal or general emotional intensity or valence (Walter et al., 2008a). While the ventral striatum and hypothalamus were identified as core structures of sexual arousal, the pregenual anterior cingulate cortex 
(pgACC) was found to integrate information of sexual intensity and emotional valence. The role of the thalamus, however, was defined as mediating emotional intensity, mainly via activations in its mediodorsal compartment. Insufficient spatial resolution in prior studies limited interpretation of thalamic activations despite its well described functional parcellation according to a number of intriguing studies in animals. In humans, recent investigations confirmed functional and structural specificity of thalamocortical connectivities (Johansen-Berg et al., 2005; Klein et al., 2010; Zhang et al., 2010). Given the functional heterogeneity of the cortical regions, an involvement of the thalamus as a whole in one subcomponent of sexual arousal could at least be questioned, and in light of its considerably diverse connections to a number of subcortical and cortical "hubs", its role may have been underestimated or oversimplified.

In the same direction, the functional integration of distinct thalamocortical loops, and thus thalamic nuclei, into cortical and basal ganglia networks could not be considered for clinical concepts based on human imaging findings. Accordingly, controversial findings of increased or decreased functional connectivity between anterior cingulate cortex (ACC) and "the thalamus" as a whole could not be satisfactorily traced back to different thalamic target structures, but were related to differences in patient populations or methodologies (Anand et al., 2005; Greicius et al., 2007; Walter et al., 2009).

Thalamocortical loops, connecting functionally segregated, higher order cortical regions, and basal ganglia, have been proposed not only for well described motor and sensory regions, but also for limbic and prefrontal areas relevant for affective and cognitive processes (Alexander et al., 1986). The high specificity of these latter functions to humans, however, renders most invasive neuroanatomical approaches impossible and interspecies translations difficult.

Mirroring one major distinction of cortical functional networks, a thalamic set of regions mediating either cognitive attentional or affective interoceptive processing may be hypothesized. Since both of these functional networks, namely the default mode network and the task positive network, comprise of characteristic nodes in the prefrontal cortex (PFC), current parcellations of the thalamus into components with preferential connectivity to PFC, or other large cortical lobes (Zhang et al., 2010), may not adequately address this functional segregation within the thalamus. There is however strong evidence, that anatomical parcellations of the thalamus may in fact serve the purpose of functional segregation both in animals and humans.

The involvement of the mediodorsal thalamus (MD) in emotional processing and its distinct detectability by high resolution fMRI has been shown even on a single-subject level (Walter et al., 2008b). Coactivation with rostral ACC, previously coined the "affective division" of the cingulate cortex, has been reported in the context of increased emotional salience during erotic processing (Walter et al., 2008a).

The intralaminar thalamic nuclei, particularly the centromedian/ parafascicular thalamic complex $(\mathrm{CM} / \mathrm{PF})$ are involved in attention processing and general arousal including the control of the level of cortical activity (Haber and Calzavara, 2009). They provide strong projections to the dorsal anterior cingulate cortex (dACC) (van der Werf et al., 2002). The dorsal, "cognitive division" of the ACC (Devinsky et al., 1995) together with the anterior insula form the core components of both the salience network (Seeley et al., 2007) and the cingulo-opercular attention network (Dosenbach et al., 2008) which is crucial for maintaining attention to previously selected tasks or targets.

In addition to a functional segregation, the inclusion of basal ganglia, functional cortical divisions and thalamic subregions into the distinct loops has to be shown to support functional integration. The MD is one major relay nucleus in the thalamus and its putative role in connecting basal ganglia and cortex has been widely addressed invasively, however, it has been poorly substantiated using direct support from non-invasive imaging data in humans. As part of the salience network, MD was proposed to connect anterior insula and dACC, two regions which are themselves characterized by specific neuronal setup of large bipolar neurons. Besides its relation to salience that closely links MD to the processing of stimuli capable of drawing and binding our attention, this nucleus has also been related to the processing of the emotional experience that is often associated with salient stimuli, but processed in an affective network encompassing more rostral portions of ACC (Devinsky et al., 1995).

In this context, the processing of sexually salient and emotionally relevant stimuli, in combination with an attentional task, seems a perfect model to investigate the functional segregation and integration of thalamocortical networks. These networks are set up by specific thalamic hubs, residing in anatomically predefined nuclei and specifically process e.g., preceding attention, which can be used to discern cognitive and stimulusdriven components of attention to salient material (Corbetta and Shulman, 2002).

Direct comparison of functional connectivity of MD and CM/ $\mathrm{PF}$, with its putative targets in affective and cognitive divisions of the ACC and insula, has not been attempted. Therefore, our study aimed to reveal this relationship by using the high resolution of a 7 Tesla functional imaging setup, able to specify small structures on subcortical level, that are not detectable on lower fields. In addition, it was tested, if exceeding these two components of the multiple dimensions of sexual arousal, specific hubs of the other two dimensions, namely the motivational and autonomous components, could be found at an adequate spatial resolution.

\section{MATERIALS AND METHODS SUBJECTS}

We scanned 10 healthy, heterosexual male right-handed subjects (mean age: 25.6 years SD: 1.51 ). All subjects had a partner at the time of scanning, were sexually active and recent or previous sexual dysfunction was excluded in a standard clinical interview. Prior to the fMRI experiment, all subjects were further examined by an experienced neurologist. No subject had to be excluded for history of neurological or psychiatric disorders and all subjects performed within the normal range during neuropsychological assessment of individual attention and concentration performance using the d2 test of attention (Brickenkamp and Zillmer, 1998). The study was approved by the local IRB. Research subjects participated after giving informed written consent. 


\section{PARADIGM}

We adopted the stimulation paradigm described in Heinzel et al. (2006) and Walter et al. (2007, 2008b), which has been reported to reliably induce sexual and emotional arousal by means of subjective self-assessment and which was found to effectively elicit neural responses in key structures relevant for sexual and emotional arousal (Walter et al., 2007). To gain sufficient power for a singlesubject single-run paradigm, the number of stimulus repetitions was increased, extending the total duration to $13.6 \mathrm{~min}$. Picture sets consisted of 20 erotic and 20 non-erotic emotional pictures of humans, taken from the international affective picture system (IAPS) (Lang et al., 2005). Picture sets were counterbalanced for standard values of arousal, pleasantness, and dominance as provided by the IAPS. Furthermore, the categories were balanced for mean ratings of perceived emotional intensity as well as for perceived saliency, defined as the degree to which a stimulus captures a subject's attention. These were rated separately to account for possible interactions between both aspects of general arousal. Together with a measure of pleasantness and sexual intensity, the ratings were previously obtained from 22 healthy male subjects (mean age: 26.4 years SD: 4.1). The values were 14 (SD 6) for sexual intensity in emotional, and 61 (SD 13) for specific sexual intensity (SSI) in sexual pictures. There was no significant difference between erotic and non-erotic emotional pictures (mean values \pm SD) regarding the values of emotional intensity, which is a marker of general emotional arousal (GEA) $(55 \pm 13,54 \pm 12)$, salience ( $54 \pm 14$, $57 \pm 10)$, or valence $(62 \pm 10,66 \pm 9)$.

After the scanning session, our 10 subjects were asked to rate erotic and non-erotic stimuli for induced sexual arousal, emotional intensity, salience, and perceived feeling of pleasure. This was done to assure that the stimuli induced sexual arousal in our subjects and that they were matched for all categories except sexual intensity, as emotional pictures were taken to be non-erotic.

Pictures were presented for $4 \mathrm{~s}$ and were projected to a screen mounted to the head coil via a LCD projector. After each picture presentation, a white fixation cross appeared for a variable duration of 7.5-10.5 s and served as an experimental resting period. Stimuli were preceded by short presentations of arrows at durations of 3-5 s. The subjects were instructed to actively anticipate the upcoming picture category as sexual or emotional intense indicated by special types of white arrows on a black screen: Arrows either indicated the type of the subsequent picture when they were presented with an exclamation mark (erotic picture: upward, nonerotic picture: downward arrows) or provided information about the number of people on the subsequent picture (upward with one dot: one person; upward with two dots: two people). Arrows indicating numbers of subjects did not indicate whether the subsequent picture was an erotic or non-erotic emotional picture and both picture categories were equal in regards of number of displayed persons. Both erotic and emotional pictures were explicitly cued in $50 \%$ of the cases and a total of 12 arrows were presented without subsequent picture conditions. Active anticipation of the upcoming picture category was explicitly required from the subjects and was explained to be necessary for the experiment. Subjects were asked to passively view the upcoming pictures during the subsequent picture condition and let it act on them. No active response was requested to avoid confound on our experiment e.g., by motor preparation. The instruction was given that during each fixation period (indicated by a fixation cross, also shown to the subject prior to the experiment), subjects should disengage themselves from the last condition, just fixating the cross. They were explained, that pictures randomly appear, being cued or not cued by a preceding arrow and that in very rare cases, arrows could appear without subsequent picture, just being followed by a fixation cross. They were told, that this was necessary for the experimental design, but very rarely the case, so active anticipation should be performed any time. No misleading cues were used by our design, to assure subjects' compliance and all pictures were only shown once. After subjects have entered the scanner, prior to experiment, indication of arrows as well as a very short repetition of the instruction was given to the subjects and they were asked for open questions.

In our analysis, we focus on the erotic and emotional anticipation periods as well as the picture perception phase.

\section{IMAGE ACQUISITION}

All experiments were performed on a 7 Tesla whole body MR system (Siemens, Erlangen, Germany). An eight-element phased array coil (Rapid Biomedical, Germany) was used for signal transmission (RF power distributed to result in a pseudo CP excitation) and reception (eight independent receive channels). Anatomical reference data were acquired with 3D-MPRAGE ( $1 \mathrm{~mm}$ isotropic resolution, TI 1050, TR $2300 \mathrm{~ms}$, flip angle $5^{\circ}$ ). For high resolution functional imaging, single-shot EPI was optimized for 7 Tesla (see Figure 1). SAR was reduced by decreasing the nominal fat saturation flip angle. Imaging parameters were FOV $220 \times 220 \mathrm{~mm}$, matrix size $128 \times 128$, 16 slices, 3 -mm slice thickness, 0.6-mm gap, TR 1000, TE 24 ms, 6/8 partial Fourier, GRAPPA factor 2, sinusoidal readout gradient. The small voxel volumina of $12 \mu \mathrm{l}$ result in reduced

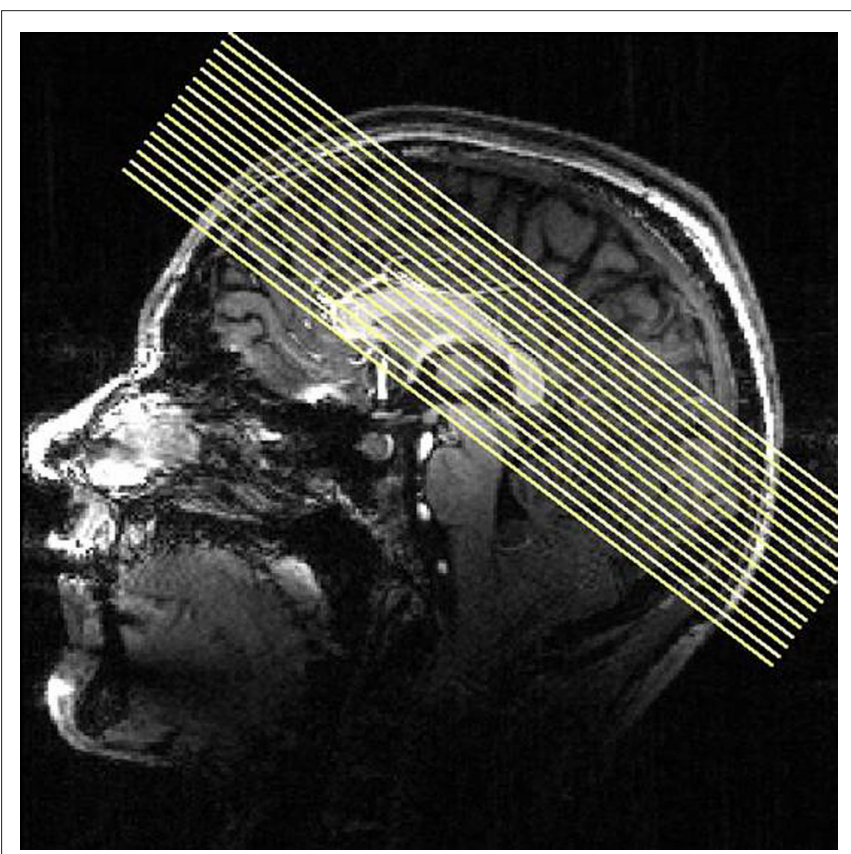

FIGURE 1 | Orientation of functional slices during fMRI session. Sixteen slices were acquired in an interleaved order. 
dephasing across the voxel. Therefore, high spatial resolution allows a minimization of signal dropouts. During the online reconstruction, all data were motion and distortion corrected based on a reference measurement of the local point spread function (Zaitsev et al., 2004), which was optimized for use in high fields (Speck et al., 2008).

\section{DATA PREPROCESSING AND ANALYSIS}

Preprocessing and statistical analysis was performed using BrainVoyager QX 1.9 (Brain Innovation, Maastricht, The Netherlands) (Goebel et al., 2006). Preprocessing of the functional scans included a more accurate offline correction of residual head motion, slice scan time correction and removal of linear trends. A high pass filter of $0.0037 \mathrm{~Hz}$ was applied, corresponding to three replication cycles or less over the whole session, to remove low frequency noise that could not be explained by our design.

Functional images were co-registered with anatomical images and resliced to 3D data sets using a trilinear interpolation algorithm. This transformation resulted in isotropic voxels of $2 \times 2 \times 2 \mathrm{~mm}$, which was found to be a reasonable trade off between spatial resolution and the number of voxelwise comparisons to correct for. Anatomical and functional data were transferred into Talairach space as implemented by the software used. Statistical analysis was performed creating individual three-dimensional statistical maps for each subject. Smoothing of $4 \mathrm{~mm}$ was applied to all data. Parameter estimates for our experimental conditions were calculated using a general linear model (GLM) (Friston et al., 1995) on $3 \mathrm{D}$ volume time courses.

The fixation period was entered as a regressor of no interest in our design matrix and was not further analyzed for the purpose of this study. The design matrix included regressors of interest for the different types of picture presentation and for the anticipation periods. Group analysis was performed using a random effects model.

Conditions and contrasts were tested separately. To control for multiple comparisons, the standard false discovery rate (FDR) (Benjamini and Hochberg, 1995; Genovese et al., 2002) method implemented in BrainVoyager QX was used for orienting contrasts. This thresholding method computes a single voxel threshold for the desired level of false positives according to the number of detected suprathreshold voxels. The FDR was set to $q<0.05$, which means that less than $5 \%$ of all voxels were accepted to be false positives.

Subsequent statistical analyses for thalamic subregions MD and CM are reported on an (uncorrected) p-threshold of 0.001 . Complex conjunction analyses were calculated for a $p<0.05$, using the conjunction null approach. This method tests for voxels in which both contrasts included in the conjunction yield significant results reflecting the assumptions of a logical conjunction. This is preferred over the global null conjunction testing against the case that both contrasts are non-significant (Nichols et al., 2005).

Resulting 3D statistical maps were then overlaid on subjects' anatomical high resolution images to relate activations to underlying structures which were identified using a standard anatomical atlas (Mai et al., 2004). Group results are displayed on a high resolution template provided by the MRIcron software (Rorden et al., 2007), which was transferred into Talairach space. Effects were further validated by direct overlays on the original EPI data to account for possible susceptibility artifacts from neighboring structures. Following a standard protocol in BrainVoyager QX for anatomical overlays, statistical maps were interpolated to correspond to the underlying anatomical resolution of $1 \mathrm{~mm}^{3}$.

Subjective ratings of sexual intensity, emotional intensity, and arousal were assessed on a visual analog scale from 10 to 90 (Walter et al., 2008a). Differences between emotional and erotic picture conditions in all three subjective dimensions were compared using a two-sided paired $t$-tests with a significance level of $p<0.05$.

\section{RESULTS \\ BEHAVIORAL ASSESSMENT}

To test the matching of emotional dimensions in our subjects, we directly compared their ratings of GEA, SSI, and emotional valence for both erotic and emotional stimuli. Comparing emotional/erotic stimuli, the mean values \pm SD were $60 \pm 13 / 56 \pm 12$ for GEA, $56 \pm 13 / 58 \pm 9$ for salience, $14 \pm 5 / 62 \pm 16$ for SSI and $69 \pm 11 / 65 \pm 9$ for valence. While erotic and non-erotic emotional stimuli did not differ in subjective ratings of GEA, salience and valence ratings $(p>0.2)$, there was a significant difference of their SSI ratings $(t=-9.319, p<0.0001)$.

\section{SEGREGATION OF GENERAL EFFECTS OF PICTURE VIEWING AND PICTURE EXPECTANCY}

To differentiate between networks related to attentional and perceptive task components, anticipation (representing a rather attentional task), and picture viewing (representing a more perceptive task) were compared: Anticipation of both erotic and emotional pictures revealed overlapping thalamic activations in bilateral CM/ $\mathrm{PF}$, superior colliculus and pulvinar by the conjunction analysis [expectancy of erotic $\times$ expectancy of emotional]. The effects of anticipation were restricted to intralaminar portions of the thalamus even at the uncorrected conjoint threshold of $p<0.05$ (see Figure 2, blue voxels).

In contrast, the conjunction of main effects of picture viewing [erotic pictures $\times$ emotional pictures] revealed common effects in the bilateral MD formation. However, these effects did not extend into the intralaminar portions, covered by the first conjunction (see Figure 2, red voxels). Similar to the expectancy condition, additional subcortical activations for main effects of picture viewing were found in the bilateral pulvinar, superior colliculus and in the right putamen ( $p<0.05$ for conjunction).

This distinct assignment of CM/PF to anticipation and MD to visual perception of erotic and emotional stimuli was also reflected by coactivation of characteristic cortical networks of the respective main effects of expectancy or picture viewing: Anticipation was related to bilateral activations in the dorsal ACC, supracallosal midcingulate cortex (MCC), posterior cingulate cortex (PCC), and anterior insula (see Figure 3) as well as (bi-)lateral supramarginal gyrus (SMG), medial temporal complex $(\mathrm{MT}+)$, superior temporal sulcus (STS), visual cortex and activation in right medial frontal gyrus (MFG), superior frontal sulcus (SFS), inferior frontal sulcus (IFS), and superior temporal gyrus (STG). Picture viewing instead was found to show common activations in the visual cortex and supracallosal MCC as well as left insular cortex, bilateral inferior frontal gyrus (IFG), MFG, and precentral gyrus (PrecG, see Table 4). 


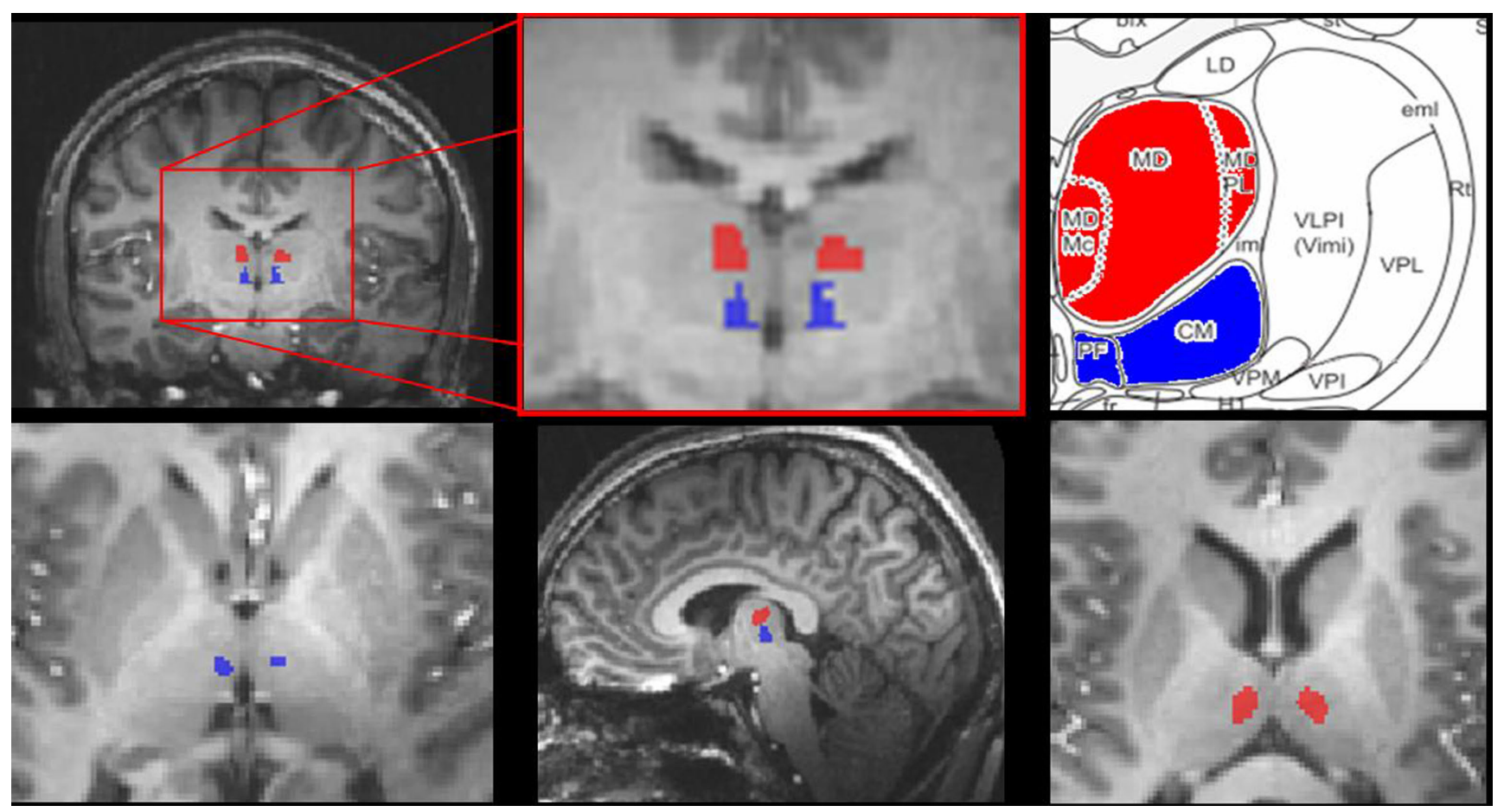

FIGURE 2 |Thalamic activation during anticipation and picture period. Thalamic activation for the conjunction [erotic pictures $\times$ emotional pictures] revealed the bilateral mediodorsal thalamus (red voxels) while the anticipation period, examined by the conjunction [erotic anticipation $\times$ emotional anticipation], activated the intralaminar centromedian/parafascicular thalamic nucleus (blue voxels) (conj. $p<0.05, x:-5, y .-13, z$ : 10, right; $z$ : 3, left). Right upper figure adapted from Mai, J., Assheuer, J., and Paxinos, G. (2004). Atlas of the Human Brain. San Diego: Academic Press/Elsevier.

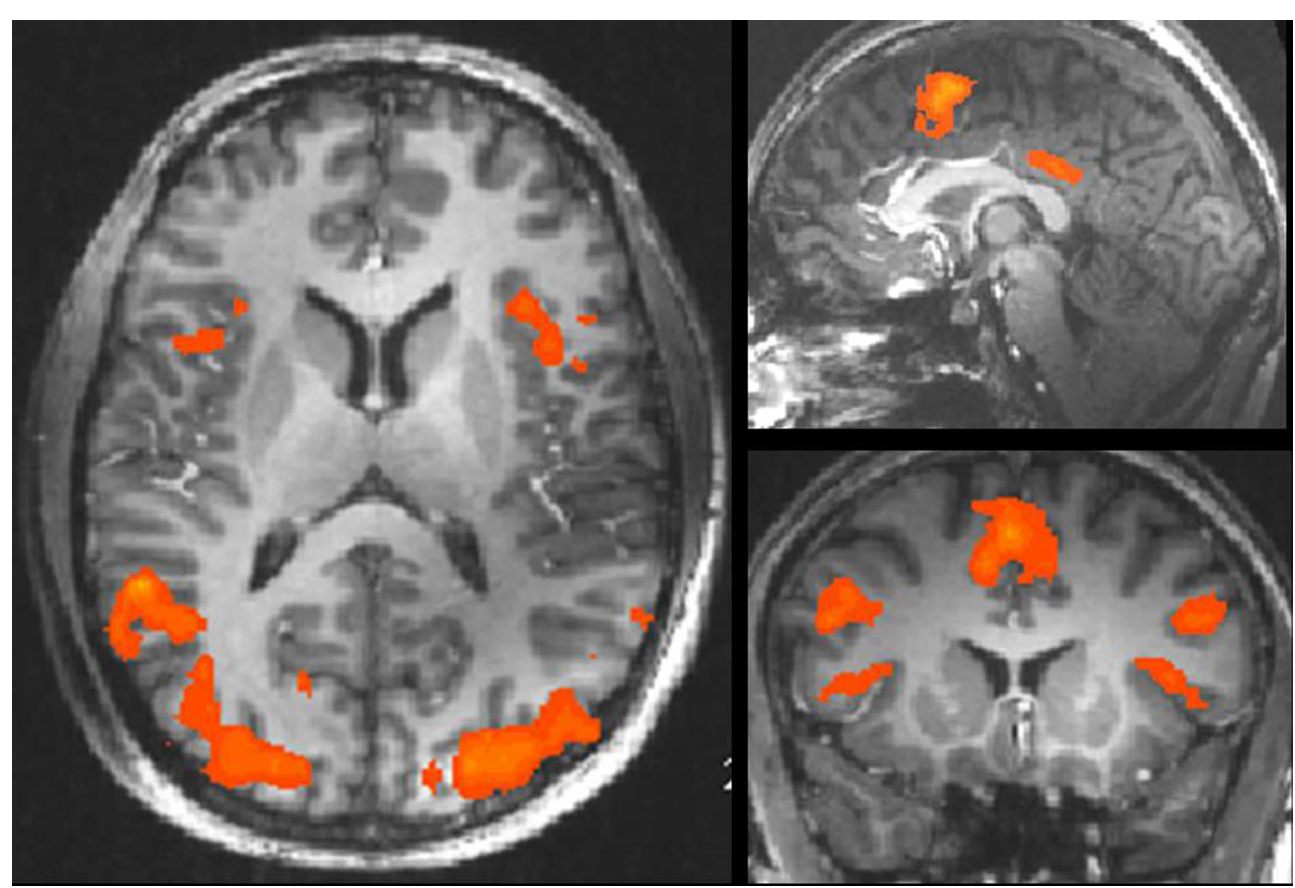

FIGURE 3 | Cortical activation during anticipation period. Regions with significant signal increases during anticipation period [erotic anticipation $\times$ emotional anticipation] (conj. $p<0.05$ uncorrected, $x:-1, y .1, z$. -10 ; see also Table 2). 


\section{MAIN EFFECT OF SEXUAL AROUSAL IN SUBCORTICAL STRUCTURES}

Sex-specific effects were defined as those effects that were task independent and irrespective of GEA. They were revealed by the conjunction of contrasts between sexual and emotional conditions of both tasks [anticipation of sexual picture $>$ anticipation of emotional picture $] \times$ [sexual picture $>$ emotional picture] (at a conjoint threshold of $p<0.05$ uncorrected). Main effects of sex were found on subcortical level in the right paraventricular portion of the thalamus (Figure 4), reflecting the overlapping effect of sex on anticipation and picture period (including the paraventricular mediodorsal, laterodorsal (LD), and parataenial (PT) thalamic nuclei), and the right head of caudate nucleus.

Cortical activation affected the bilateral inferior parietal sulcus (IPS), medial temporal gyrus (MTG), right superior parietal lobule (SPL) as well as left SMG, postcentral gyrus, and occipital gyrus (Table 3). It is noteworthy that we did not find general effects in the main regions of specific effects of either expectancy or picture conditions with the exception of the paraventricular thalamus. Medial prefrontal and cingulate cortex did not show overlapping effects of sex neither in expectancy nor in picture condition. We also did not find general effects of sexual intensity in insular cortex as far as it was covered by our investigation.

\section{SPECIFIC EFFECTS OF SEX WITHIN EXPECTANCY AND PICTURE PERIODS Erotic versus emotional anticipation}

To analyze the sex-specific, task dependent effect within the anticipation period, anticipation of erotic and emotional pictures was analyzed by direct comparison: The contrast between anticipation of erotic versus anticipation of emotional stimuli (see Table 2) elicited significantly greater thalamic activation for the erotic condition $(p<0.001)$ in the dorsal intralaminar portion with peak activations in the lateral habenula and medial centromedian complex, posterior to the PF.

Further significant activations of specific thalamic nuclei were found in the bilateral ventral anterior (VA) thalamic nucleus (Figure 5) as well as in the paraventricular portion of the thalamus, corresponding to paraventricular mediodorsal, LD, and PT thalamic nuclei. In addition, we found increased activation in the right head of caudate nucleus, left pallidum, and right putamen upon anticipation of erotic stimuli.

On the cortical level, increased activations during expectancy of erotic stimuli were found in the right anterior insular cortex and right dACC. In addition to the regions that were already revealed by the conjunction analysis of general effects of expectancy, increased activations for only sexual expectancy were found in the right frontal eye field (FEF), the bilateral IPS, STS, MT+, and left SPL (see Table 2).

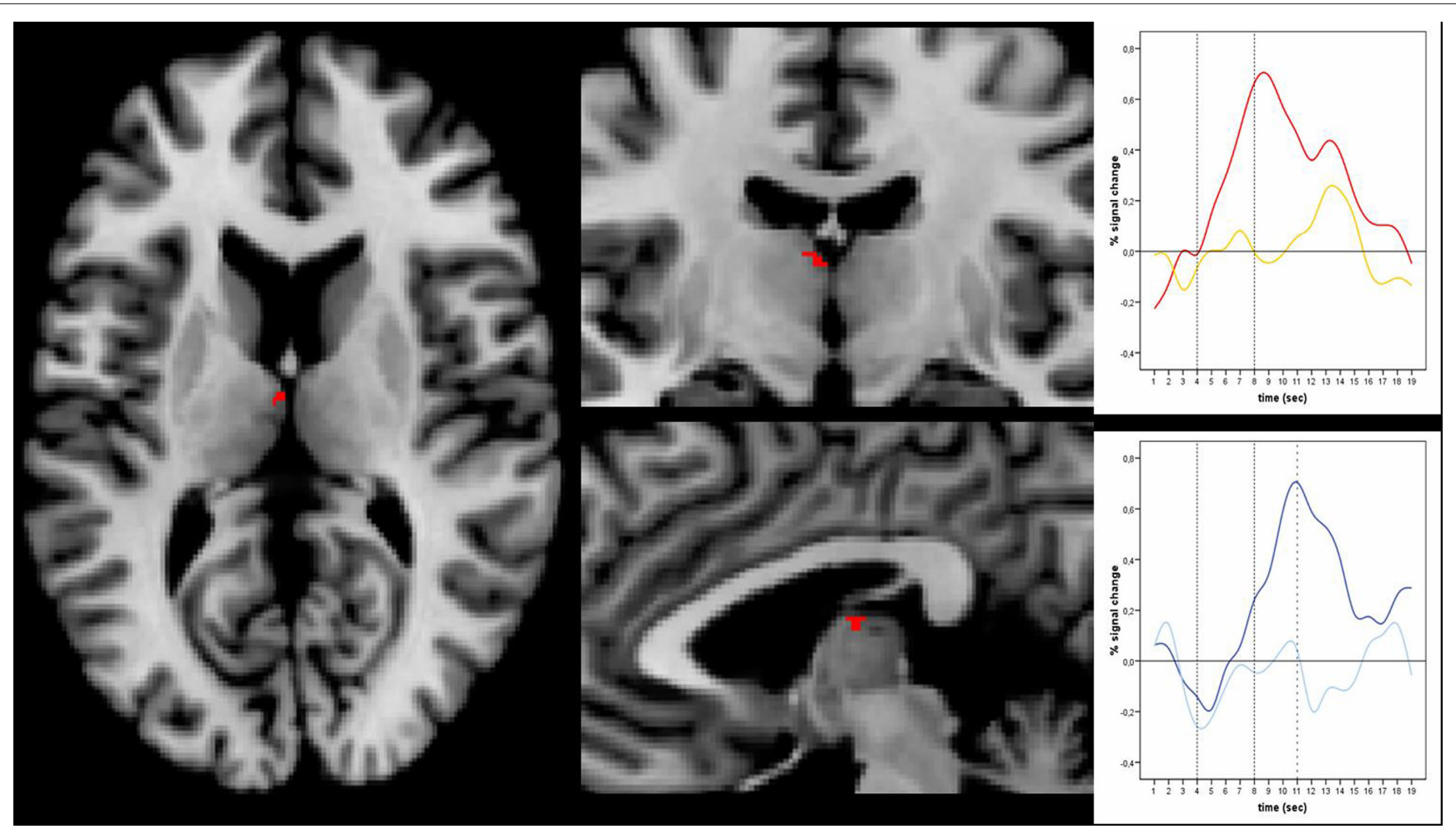

FIGURE 4 | Main effects of sex. Left and middle: Main effects of sex in the right paraventricular thalamus including laterodorsal, parataenial, and paraventricular mediodorsal thalamic nucleus was shown by the conjunction [anticipation of erotic pictures $>$ anticipation of emotional pictures] $\times$ [erotic pictures $>$ emotional pictures] at an uncorrected threshold of conj. $p<0.05$ ( $x: 3 ; y .-13 ; z: 12$ ). Right: Timecourses in the paraventricular thalamus shown separately in the upper picture for erotic (red) and emotional picture perception (yellow) and in the lower picture for erotic (dark blue) and emotional anticipation (light blue). Peaks for erotic expectancy conditions lagged those of the erotic picture conditions by $3 \mathrm{~s}$, which was the actual lag of expectancy and subsequent picture conditions. This indicates at least a confound on the expectancy effects by their subsequent picture conditions. 
Table 1 | Table of abbreviations.

\begin{tabular}{|c|c|}
\hline ACC & anterior cingulate cortex \\
\hline alfO & anterior insula, frontal operculum \\
\hline Cd_head & head of caudate nucleus \\
\hline $\mathrm{CM} / \mathrm{PF}$ & centromedian/parafascicular thalamic complex \\
\hline dACC & dorsal anterior cingulate cortex \\
\hline FEF & frontal eye field \\
\hline IFG & inferior frontal gyrus \\
\hline IFS & inferior frontal sulcus \\
\hline Insula & insular cortex \\
\hline IPS & inferior parietal sulcus \\
\hline LD & laterodorsal thalamic nucleus \\
\hline $\mathrm{LHb}$ & lateral habenula \\
\hline MCC & midcingulate cortex \\
\hline MD & mediodorsal thalamic nucleus \\
\hline MFG & medial frontal gyrus \\
\hline MT+ & medial temporal complex \\
\hline MTG & medial temporal gyrus \\
\hline NclCaud & caudate nucleus \\
\hline OcG & occipital gyrus \\
\hline Pall & globus pallidus \\
\hline parav. Thal & paraventricular thalamus \\
\hline PCC & posterior cingulate cortex \\
\hline pgACC & pregenual anterior cingulate cortex \\
\hline PoCG & postcentral gyrus \\
\hline PrecG & precentral gyrus \\
\hline Precun & precuneus \\
\hline PT & parataenial thalamic nucleus \\
\hline Put & putamen \\
\hline SFS & superior frontal gyrus \\
\hline SMA_lat & supplementary motor area (lateral) \\
\hline SMG & supramarginal gyrus \\
\hline SPL & superior parietal lobule \\
\hline STG & superior temporal gyrus \\
\hline Str. term. & Stria terminalis \\
\hline STS & superior temporal sulcus \\
\hline VA & ventral anterior thalamic nucleus \\
\hline
\end{tabular}

\section{Picture period}

To identify the effects of sex and emotion on the perception phase, activity during erotic, and emotional picture viewing were analyzed separately in orienting contrasts for erotic and emotional picture periods:

We found distinct significant effects of erotic picture viewing and emotional picture viewing in the mediodorsal thalamic nucleus $(q<0.05$, FDR corrected $)$.

However the peak activations differed between picture conditions with sexual picture periods leading to peaks which were located more mediodorsally, while emotional pictures led to activations in the (bi-)lateral portions of MD (see Figure 6).

Additional activations for erotic picture presentation were located in the right anterior thalamus and pulvinar, the bilateral head of caudate nucleus, tectum, right putamen, and left claustrum $(q<0.05$, FDR corrected), while emotional picture viewing only showed effects in bilateral pulvinar, tectum and putamen, but not in claustrum and caudate nucleus.
The conjunction analysis of general effects of picture viewing further revealed a cortical effect in the mid-insular cortex. However, this effect was left lateralized for erotic and more dorsally and right lateralized for emotional picture conditions.

Additionally, both erotic and emotional picture conditions elicited significant signal increases in the supracallosal anterior and MCC.

Other effects were found in the MFG, IFS, precentral gyrus (PrecG), postcentral gyrus, precuneus (Precun), SFS, SMG, STG, STS, MTG as well as primary, and secondary visually areas (FDR, $q<0.05$, see also Table 4 ).

\section{Erotic versus non-erotic emotional picture perception}

The effect of sex on the perception phase was analyzed by direct comparison of erotic and emotional picture viewing, the latter matched for emotional intensity and number of displayed people. We thus extracted the sex-specific effect independent of emotional intensity or general processing of human figures.

The contrast revealing greater activations during sexual conditions than during emotional picture periods showed main subcortical effects in the right paraventricular portion of the thalamus (corresponding to paraventricular mediodorsal thalamus, LD, and PT thalamic nucleus), the bilateral head of caudate nucleus and the stria terminalis $(p<0.001$ uncorrected). Cortical effects were located in the right PCC, left precuneus, bilateral IPS, and MTG as well as left precentral gyrus ( $p<0.001$, see also Table 4$)$.

\section{GREATER EFFECTS OF SEX DURING ANTICIPATION OR PICTURE PERIODS}

We compared the sex effects during anticipation and picture period to identify those sex-specific effects that were task dependent. A taskby-stimulus interaction was found with greater effects of sex during expectancy conditions than during picture periods: The contrast [anticipation of erotic pictures $>$ anticipation of emotional pictures] $>$ [erotic pictures $>$ emotional pictures] revealed subcortical effects only in the right dorsal intralaminar portion with peak activations in the lateral habenula and medial centromedian thalamic complex, posterior to the $\mathrm{PF}$ - the same region that was also shown for the effects of sex on the anticipation period itself $(p<0.001$ uncorrected). The only cortical structures that showed greater effect of sex on the expectancy condition were the bilateral anterior insular cortex, dACC, SMG, STS, and left precentral gyrus (see Table 5).

Except one cluster in right IPS, we did not find regions that showed greater influence of sex on the picture conditions: No other significant effect was found for the inverse comparison of erotic versus emotional effects in both conditions by the contrast [erotic pictures $>$ emotional pictures] $>$ [anticipation of erotic pictures $>$ anticipation of emotional pictures] for an uncorrected threshold of $p<0.001$.

\section{DISCUSSION}

Extending prior results in single subjects (Walter et al., 2008b) on a group level, we were able to detect subcortical thalamic activation restricted to single nuclei using a single-run design with an optimized high resolution single-shot EPI acquisition method at 7 Tesla.

\section{SUBCORTICAL FINDINGS}

Our data confirm, for the first time, activations of distinct thalamic nuclei that are restricted to their respective anatomical boundaries and specific to their proposed function. Therefore, 


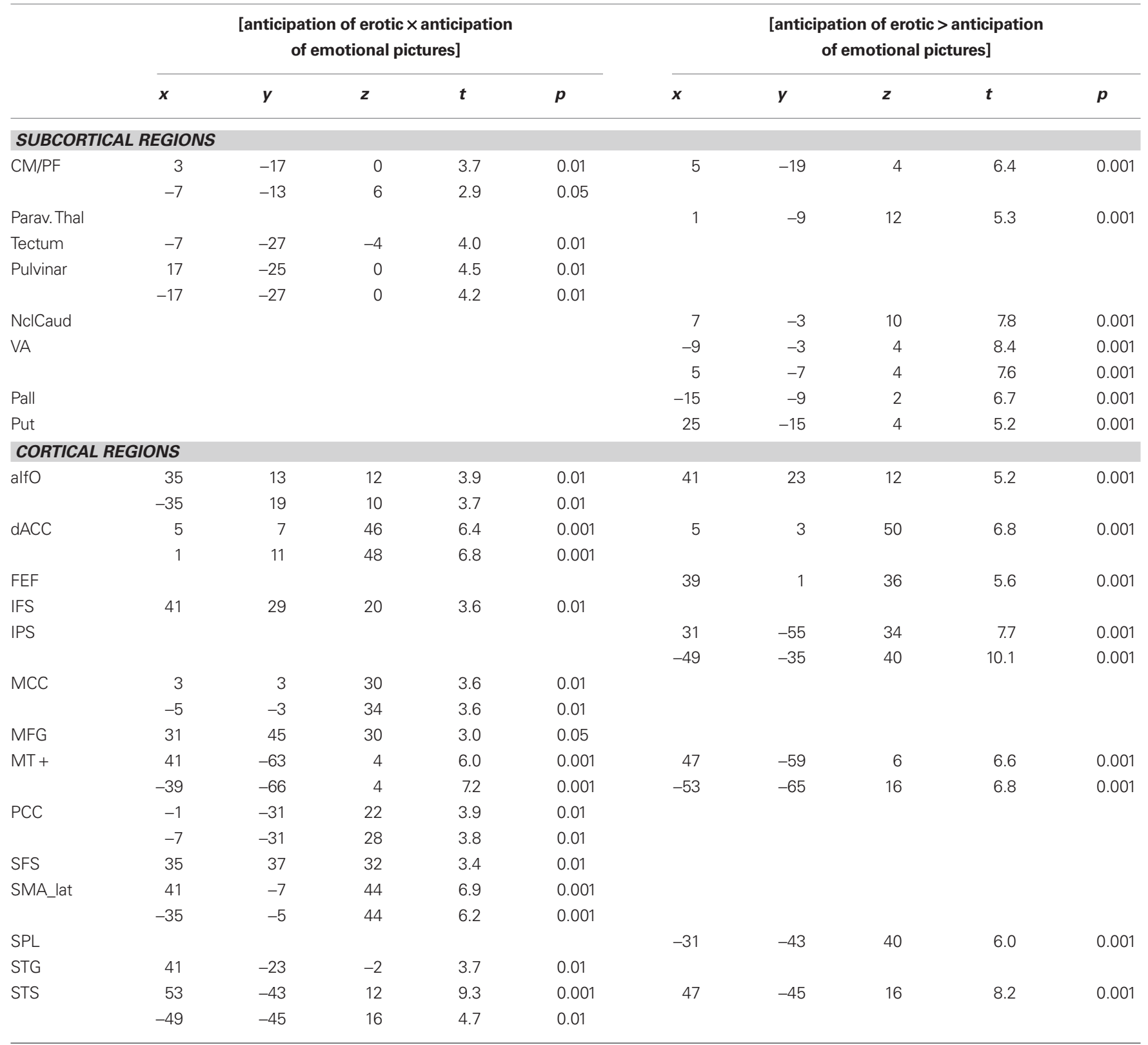

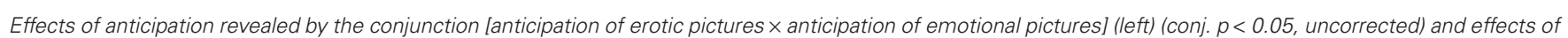
sexual arousal during anticipation period revealed by the contrast [anticipation of erotic pictures $>$ anticipation of emotional pictures] (right) ( $p<0.001$, uncorrected, $x, y, z$ coordinates in Talairach space). For abbreviations see Table 1.

our findings lend support to the theory of functional subdivisions within the thalamus coactivating with distinct basal ganglia and cingular as well as insular subregions and thus support the distinction of affective and cognitive cortical subdivisions, based on segregated thalamocortical loops (Devinsky et al., 1995; Bush et al., 2000).

According to our findings, activations in the mediodorsal thalamic nucleus can be primarily related to the emotional intensity of a stimulus while the centromedian/parafascicular complex is rather affected by general attentional processing, irrespective of the emotional context. In contrast, the paraventricular mediodorsal complex, including parts of the LD and PT thalamic nucleus can be related to stimulus-specific sexual content independent of the current task, i.e., expectation or picture perception. These sexspecific activations were mirrored correspondingly by a main effect of sexual intensity in the head of caudate nucleus. Interactions in terms of task dependent effects of sex that appeared specifically during anticipation periods were limited to posterior intralaminar and habenula portions of medial thalamic regions. This suggested functional distinction is well in line with a number of invasive studies in animals and serves as an indirect evidence for noninvasive studies in humans. 


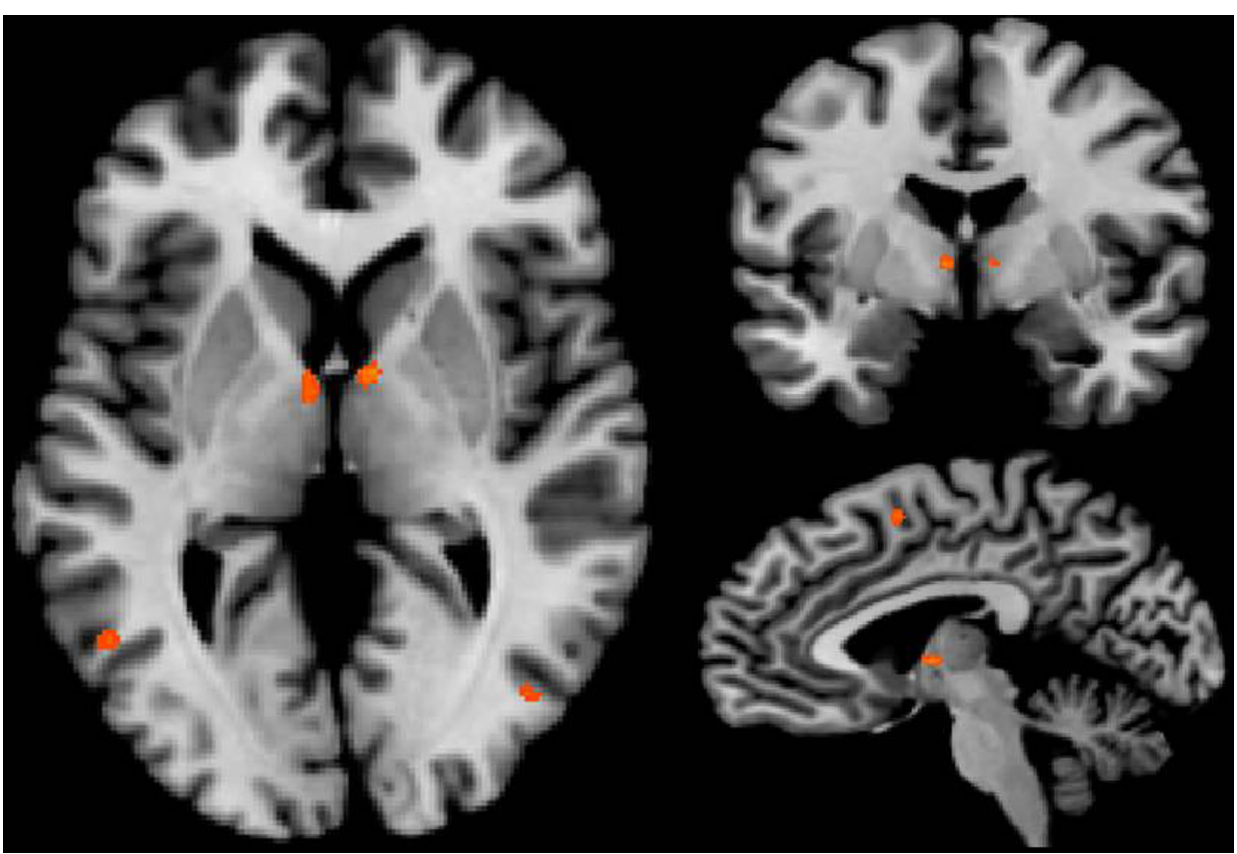

FIGURE 5 | Bilateral activation in the ventral anterior thalamic nucleus was revealed by the contrast [anticipation of erotic pictures $>$ anticipation of emotional pictures] at an uncorrected threshold of $p<0.001, x: 4 ; y:-6 ; z: 5$.

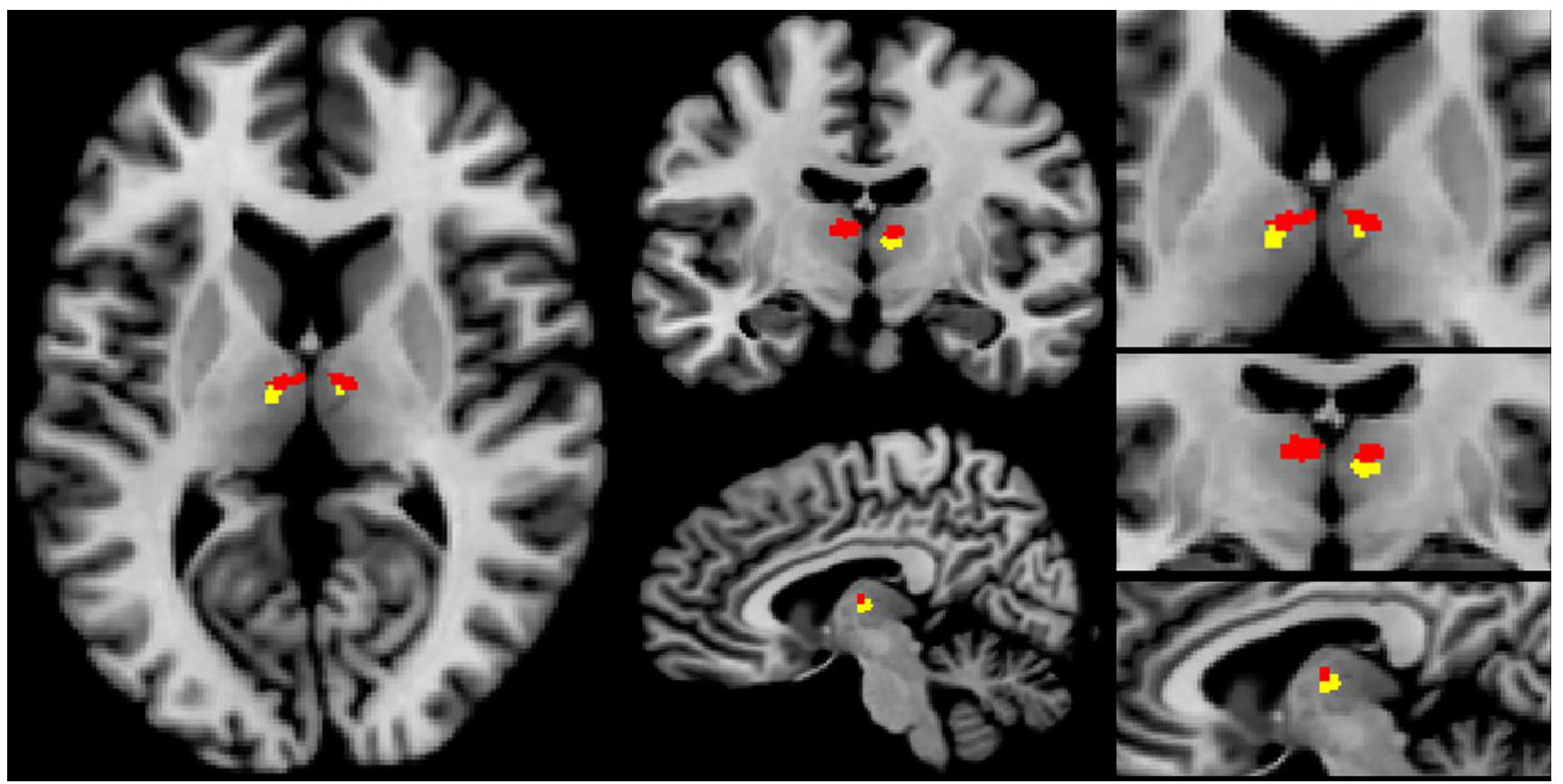

FIGURE 6 |Thalamic activation for erotic and emotional picture perception in the bilateral mediodorsal thalamic nucleus. Erotic picture perception showed a more mediodorsal activation pattern (red voxels), while activation for emotional picture perception was located more laterally (yellow voxels) ( $p<0.001$, uncorrected, $x:-7, y:-13, z: 10)$.

Intralaminar activations: the centromedian/parafascicular complex is involved in attention processing

The centromedian/parafascicular thalamic complex was previously found in animals to be related to attentional processing (Kinomura et al., 1996; Haber and Calzavara, 2009) and attentional shift to salient stimuli (van der Werf et al., 2002). It was shown to be part of the ascending reticulo-thalamo-cortical activating system (Moruzzi and Magoun, 1949; Isaacson and Tanaka, 1986; Cornwall 
and Phillipson, 1988b) and to be involved in sexual arousal and erection in rats and gerbils (Heeb and Yahr, 1996, 2001; Coolen et al., 1997, 2003a,b; Veening and Coolen, 1998; Temel et al., 2004).

As shown by Matsumoto and colleagues, activations in macaque $\mathrm{CM} / \mathrm{PF}$ neurons were not responsive to reward but to unexpected stimuli when investigated with electrophysiological recordings.

Table 3 | Main effects of sexual arousal.

\begin{tabular}{lrrrll}
\hline & \multicolumn{1}{l}{$\boldsymbol{x}$} & $\boldsymbol{z}$ & $\boldsymbol{t}$ & $\boldsymbol{p}$ \\
\hline Cd_head & 11 & 1 & 14 & 3.9 & 0.01 \\
IPS & 31 & -53 & 34 & 3.4 & 0.01 \\
& -21 & -61 & 34 & 3.4 & 0.01 \\
PoCG & -43 & -33 & 42 & 4.2 & 0.01 \\
SMG & -51 & -33 & 40 & 3.4 & 0.01 \\
SPL & 25 & -73 & 28 & 3.8 & 0.01 \\
MTG & 39 & -71 & 4 & 3.0 & 0.05 \\
& -51 & -67 & 4 & 3.6 & 0.001 \\
OcG & -39 & -65 & -12 & 3.2 & 0.05 \\
Parav. Thal & 3 & -13 & 12 & 3.0 & 0.05
\end{tabular}

Main effects of sexual arousal revealed by the conjunction [lanticipation of erotic picture $>$ anticipation of emotional picture) $\times$ (erotic picture $>$ emotional picture)] at a conjoint threshold of $p<0.05$ ( $x, y, z$ coordinates in Talairach space). For abbreviations see Table 1 .
Their pharmacological inactivation via muscimole, however, also abolished reward related processing which was specifically related to tonic activations in striatum (Matsumoto et al., 2001). Similarly, our results suggest the involvement of these intralaminar nuclei in general attentional processes, which are not specific to sexual processing but may be regarded necessary to reorient attention toward these specifically salient stimuli.

Activations in the CM/PF portions were paralleled by coactivations in anterior insula cortex and dorsal ACC in our study, and in these regions activations were greatest for erotic anticipation. For $\mathrm{MD}$, on the other hand, no such indication of functional connectivity, in terms of coactivation during a certain condition (Friston, 1994), with the cingulo-opercular network could be found. In the attentional task, $\mathrm{CM} / \mathrm{PF}$, on the contrary, was coactive with those cortical regions with largest evidence for involvement in attentional processes: The dorsal ACC as part of the cognitive division of ACC (Devinsky et al., 1995; Bush et al., 2000) has been found in a number of studies including executive control and direction of attention. Together with the anterior insula, it has thus been proposed to form the so called attention set network (Dosenbach et al., 2008). In our study, the expectancy condition requires subjects to direct their attention to the content of the subsequent picture period. This task is likely to set attention to the upcoming event. The activation of the anterior insula during this task supports the

Table 4 | Effects of picture viewing.

\begin{tabular}{|c|c|c|c|c|c|c|c|c|c|c|}
\hline & \multicolumn{5}{|c|}{ [erotic pictures $\times$ emotional pictures] } & \multicolumn{5}{|c|}{ [erotic pictures > emotional pictures] } \\
\hline \multicolumn{11}{|c|}{ SUBCORTICAL REGIONS } \\
\hline Cd_head & & & & & & -9 & -5 & 14 & 6.6 & 0.001 \\
\hline \multirow[t]{2}{*}{ MD } & 9 & -17 & 14 & 4.3 & 0.01 & 3 & -11 & 16 & 5.9 & 0.001 \\
\hline & -7 & -13 & 8 & 4.2 & 0.01 & & & & & \\
\hline Pulvinar & -23 & -29 & 2 & 11.8 & 0.001 & & & & & \\
\hline Putamen & 19 & -1 & 10 & 3.2 & 0.05 & & & & & \\
\hline Str. term. & & & & & & 1 & -3 & 4 & 7.9 & 0.001 \\
\hline Tectum & -3 & -25 & 0 & 4.3 & 0.01 & & & & & \\
\hline \multicolumn{11}{|c|}{ CORTICAL REGIONS } \\
\hline IPS & & & & & & -41 & -35 & 40 & 9.5 & 0.001 \\
\hline MCC & -3 & 1 & 36 & 3.2 & 0.05 & & & & & \\
\hline \multirow[t]{2}{*}{ MFG } & 37 & 17 & 26 & 4.6 & 0.01 & & & & & \\
\hline & -53 & 9 & 28 & 3.2 & 0.05 & & & & & \\
\hline \multirow[t]{2}{*}{ MTG } & & & & & & 51 & -61 & 0 & 5.8 & 0.001 \\
\hline & & & & & & -49 & -63 & 0 & 6.4 & 0.001 \\
\hline PCC & & & & & & 11 & -41 & 20 & 5.9 & 0.001 \\
\hline \multirow[t]{2}{*}{ PrecG } & 41 & -1 & 32 & 5.0 & 0.001 & & & & & \\
\hline & -35 & -7 & 44 & 5.9 & 0.001 & -25 & -11 & 46 & 5.9 & 0.001 \\
\hline Precun & & & & & & -13 & -57 & 34 & 9.3 & 0.001 \\
\hline
\end{tabular}

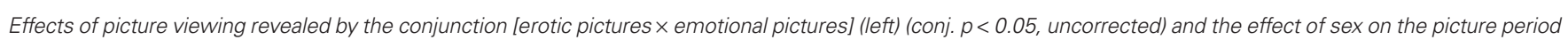
shown by the contrast [erotic pictures $>$ emotional pictures] (right) $(p<0.001$, uncorrected, $x, y, z$ coordinates in Talairach space). For abbreviations see Table 1. 
Table 5 | Comparison of effects of sexual arousal during anticipation and picture period.

\begin{tabular}{lrrrrl}
\hline & \multicolumn{1}{c}{$\boldsymbol{x}$} & $\boldsymbol{y}$ & $\boldsymbol{z}$ & $\boldsymbol{t}$ & $\boldsymbol{p}$ \\
\hline alfO & 39 & 9 & 14 & 5.2 & 0.001 \\
& -31 & 19 & 14 & 5.5 & 0.001 \\
CM/PF & 9 & -21 & 4 & 5.5 & 0.001 \\
dACC & 9 & 19 & 36 & 5.7 & 0.001 \\
& -3 & 15 & 30 & 5.3 & 0.001 \\
SMG & 49 & -39 & 38 & 7.1 & 0.001 \\
& -51 & -43 & 34 & 5.0 & 0.001 \\
STS & 49 & -41 & 14 & 5.9 & 0.001 \\
& -53 & -45 & 22 & 6.5 & 0.001 \\
PraecG & -39 & -7 & 42 & 5.9 & 0.001
\end{tabular}

Comparison of effects of sexual arousal during anticipation and picture period shown by the contrast [lanticipation of erotic pictures $>$ anticipation of emotional pictures) $>$ (erotic pictures $>$ emotional pictures)] for an uncorrected $p<0.001$. The inverse contrast [(erotic pictures $>$ emotional pictures) $>$ (anticipation of erotic pictures $>$ anticipation of emotional pictures)] did not show any significant clusters up to an uncorrected level of $p<0.001$ ( $x, y, z$ coordinates in Talairach space). For abbreviations see Table 1.

hypothesis of its involvement in attention set. Since no direct motor task is required in our design, the hypothesized role of CM/PF for mere motor adjustment, as suggested by studies in non-human primates and rodents (van der Werf et al., 2002), may need adaptation in humans. While van der Werf and colleagues proposed a differentiation of PF and CM functions based on their different projections to medial and lateral striatum, with a specific role of $\mathrm{CM}$ during sensory motor and $\mathrm{PF}$ during associative-limbic motor functions, our study cannot contribute to such a distinction in humans mainly due to insufficient resolution. We can, however, confirm their clear distinction from MD regarding its function as well as its functional connectivity.

The CM/PF has been described to be strongly connected to the basal ganglia (Royce and Mourey, 1985; Cornwall and Phillipson, 1988b; Berendse and Groenewegen, 1990; Nakano et al., 1990; Fenelon et al., 1991; Sadikot et al., 1992; Haber and Calzavara, 2009) and the motor-, premotor, and primary somatosensory cortex (Berendse and Groenewegen, 1991; François et al., 1991). These connections have been found especially for CM, while PF seems to be connected to the dACC and there especially to BA24 (Vogt et al., 1987a,b; van der Werf et al., 2002). Only few studies reported connections of CM/PF with the anterior insula in hamsters (Reep and Winans, 1982a,b) and in rhesus monkeys (Mufson and Mesulam, 1984). In humans, anatomical connections especially from CM/PF to dACC, anterior insula and to a majority of subcortical structures are supported by diffusion tensor imaging (DTI) results (Eckert et al., 2009). Our findings show a coactivation of CM/PF with dACC and the anterior insula indicating a functional connectivity of these regions under the task condition. These findings support the existence of at least a functional connectivity between CM/PF, dACC and the anterior insula also in humans.

The effect of greater activation during erotic as compared to emotional anticipation periods as found for the anterior insula, in addition to the main effect of attention, parallels results of the interaction analysis in $\mathrm{CM} / \mathrm{PF}$. While this activation was located more dorsally and may thus represent rather CM than PF activations, due to limited spatial resolution, also contributions from lateral habenular complex would be possible. In case of interpretation of the structure as lateral habenular complex, however, a negative effect of sex would need to be discussed. Neurons in the lateral habenula (LHb) inter alia respond to negative prediction errors, e.g., when an outcome of any kind is worse than expected, when positive reward is not delivered or when punishment is received. In that context, it has been strongly related to disappointment in primates and in humans (Matsumoto and Hikosaka, 2007, 2009; Salas et al., 2010). The LHb has strong connections to the ventral tegmental area (via the rostromedial tegmental nucleus) and inhibits dopamine release in this structure when activated. Besides reward modulation, the habenula has been suggested to modulate sexual, maternal and feeding behavior as well as pain (Felton et al., 1999; Klemm, 2004; Hikosaka et al., 2008; Hikosaka, 2010). On a transmitter level, it also influences the other two monoaminergic systems via direct projections to the locus coeruleus and dorsal raphe nuclei and has thus been claimed to be causally involved in depression (Sartorius and Henn, 2007; Sartorius and Meyer-Lindenberg, 2009; Sartorius et al., 2010). We find activity in the LHb complex as an interaction effect of sex during anticipation period, which is not present during picture period. Characteristics of nucleus accumbens activation potentially related to habenular activation could have served to clarify this issue. However, slice coverage did not include nucleus accumbens.

The exact attribution of the observed interaction effect thus remains open for final clarification in future studies. At this stage, it has to be noted that a task-by-sex interaction leads to significant effects during anticipation in CM but not in MD.

Investigating preceding attention toward sexually and emotional stimuli, we could show that $\mathrm{CM} / \mathrm{PF}$ is integrated into a cortical network which has previously been characterized as relevant for attention.

In contrast to this latter subcortical network mainly involved in attentional processes related to erotic processing, e.g., to direct attention and orchestrate occulomotor responses, we could confirm the role of mediodorsal thalamus for the processing of emotional tone during actual erotic stimulation (Walter et al., 2008a,b).

\section{Specific thalamic nuclei: the mediodorsal thalamic nucleus mediates emotional salience of stimuli}

The mediodorsal thalamic nucleus forms a main part of the limbic thalamus (Vogt et al., 1987a,b). Activation in this nucleus was described according to processing of emotional stimuli (Oyoshi et al., 1996; Price et al., 1996; Price, 1999; Vertes, 2006) and it seems to be impaired in mood disorders like depression (Drevets et al., 1992). It was, moreover, described as part of the salience network (Seeley et al., 2007). Despite insufficient spatial resolution, peak activations during erotic stimulation have been reported in MD (Redouté et al., 2000; Arnow et al., 2002; Karama et al., 2002; Heinzel et al., 2006). However, a specific component analysis revealed that these activations were rather caused by the emotional content of sexual stimuli, which often increases during erotic stimulation, than by the specific erotic intensity itself (Walter et al., 2008a).

Activation of the mediodorsal thalamic nucleus was found in our study during both, emotional and erotic picture perception. This activation supports the involvement of MD in emotional processing. In contrast to other studies, no differential effect between erotic 
and non-erotic picture perception was found in MD in our study. We attribute this to the specific picture set used in our study, which, based on our previous investigations, was purposely matched for GEA. Consequently, since the two picture samples did not differ in their emotional intensity ratings, no significant differential effect was expected for MD during the picture conditions.

This finding supports the hypothesis that MD strongly detects the emotional content of a stimulus and thus provides a necessary component for sexual processing. Therefore the MD is not considered a specific but rather a supportive sexual core region. This would explain the observation of sexual dysfunction after MD lesions and underlines the importance of the affective component during a multidimensional processing of sexual arousal (Redouté et al., 2000; Temel et al., 2004).

The MD was described as a relay nucleus of the thalamus, linking basal ganglia and cortex (McFarland and Haber, 2002). Subcortical connections of MD were reported to periaqueductal gray, ventral tegmental area, and claustrum in monkeys (Erickson et al., 2004) as well as to amygdala, area innominata, LHb, lateral hypothalamus, the ventral tegmental area, and the dorsal tegmental gray in rats (Krettek and Price, 1977; Cornwall and Phillipson, 1988a). Main cortical projections were found to the PFC in humans, primates and cats (Alexander and Fuster, 1973; Vogt et al., 1987a,b; Price, 1999; Erickson and Lewis, 2004). Reciprocal connections to the anterior insula have been described in rats (Allen et al., 1991), hamsters (Reep and Winans, 1982a,b), and in monkeys (Ray and Price, 1992, 1993). Functional connectivity of MD with the anterior insula has also been shown in humans (Seeley et al., 2007) and anatomical studies in vivo, using DTI, showed MD connections to anterior insula and PFC (Eckert et al., 2009; Klein et al., 2010; Zhang et al., 2010).

In this study, we found a coactivation of the supracallosal and MCC and MD thalamus during picture perception. These activations were paralleled by mid-insular activations, though it has to be noted that most ventral aspects of the anterior insula were not covered by our functional acquisition. This coactivation is in line with the findings by Seeley and colleagues which suggest an involvement of MD in salience processing. Other than task- or goal-directed attention, salience describes a stimulus property to result in reallocation and binding of attentional resources to itself. In response to an external stimulus, this process was shown to be mediated by exactly these regions, namely the supracallosal dACC and midcingulate as well as anterior insula together with peak activations most likely to represent mediodorsal thalamus (Seeley et al., 2007). While this network was best described using an independent component approach on resting-state data, we were able to replicate this network using our task data. Interestingly, we did not find significant differences during the actual picture conditions, suggesting comparable salience of emotionally matched stimuli after they have both been announced by expectancy cues. MD activations may thus be equally related to the emotional tone of the pictures or to their (comparable) salience. This is also be indicated by similar BOLD responses in anterior insula and supracallosal ACC for both conditions, which have recently been shown to specifically code salience processing (Litt et al., 2010). At the current stage, the involvement of MD in salience processing or emotional processing during erotic visual stimulation cannot be ultimately clarified by our results. The absence of clear effects of salience during erotic picture conditions, however, should be interpreted with caution given our specific experimental design using cued conditions. It has to be noted that our paradigm uses expectancy conditions to induce preceding attention toward upcoming stimuli. This restricts the interpretation of attention effects to a rather cognitive construct of attention and may not be confused with other concepts of stimulus-driven attention. Nonetheless, the work in animals to which we related our findings in $C M / P F$, was mainly done on tasks which rather involve the latter type of attention. Despite these critical limitations to our findings, it can still be stated that MD activations, in contrast to $\mathrm{CM} / \mathrm{PF}$ activations are more related to processing of aspects that lead to allocation of attention (such as emotion or salience), than to processing of attention itself (Figure 7).

\section{Laterodorsal and parataenial thalamic nucleus are specific to the task independent effect of sexual arousal}

An effect of stimulus value has to be considered for erotic stimuli. It was expected in thalamic regions that are connected to structures that have previously been reported to be associated with SSI or stimulus value such as the case for hypothalamus and ventral striatum (Walter et al., 2008a).

Laterodorsal thalamic nucleus. We could show that thalamic activations that were located at anatomical locations of LD and PT were specific to sexual processing as ascertained by the analysis of the main effects of sex. The activation of the LD thalamus specific to visual erotic stimulation that we detected in our study is supported by several findings on connectivity of LD to the limbic cortex (Thompson and Robertson, 1987; van Groen and Wyss, 1992; Shibata, 2000) and to the lateral hypothalamus (Ryszka and Heger, 1979) found specifically active during erotic perception (Walter et al., 2008a) and erotic stimulation (Georgiadis et al., 2010).

Parataenial thalamic nucleus. The PT nucleus belongs to the medial thalamic nuclei group (Rose and Woolsey, 1949).

Its projections to the nucleus accumbens (Powell and Cowan, 1954; Berendse and Groenewegen, 1991) are in accordance with the activation of the reward system found in most studies investigating sexual processing (Walter et al., 2008a). The activation of the PT nucleus specific to the perception of sexual stimuli as found in our study supports these previous findings.

It is worth noting that the analysis of the peristimulus time histograms in PT revealed that the peaks for erotic expectancy conditions lagged those for the erotic picture conditions by $3 \mathrm{~s}$, which was the actual lag of expectancy and subsequent picture conditions (Figure 4). This indicates at least a confound on the expectancy effects by their subsequent picture conditions. According to these results, activation in PT is rather caused by sexual perception than by sexual attention although an influence of sexual attention on this area is likely, but cannot fully be explained by our results.

\section{Ventral anterior thalamic nucleus and motor preparation}

A greater role in motor preparation is hypothesized for VA, which showed effects of anticipation similar to the intralaminar CM/PF complex. Different from the latter nuclei, we found stronger activations during erotic as compared to emotional expectancies and no main effect of expectancy. 


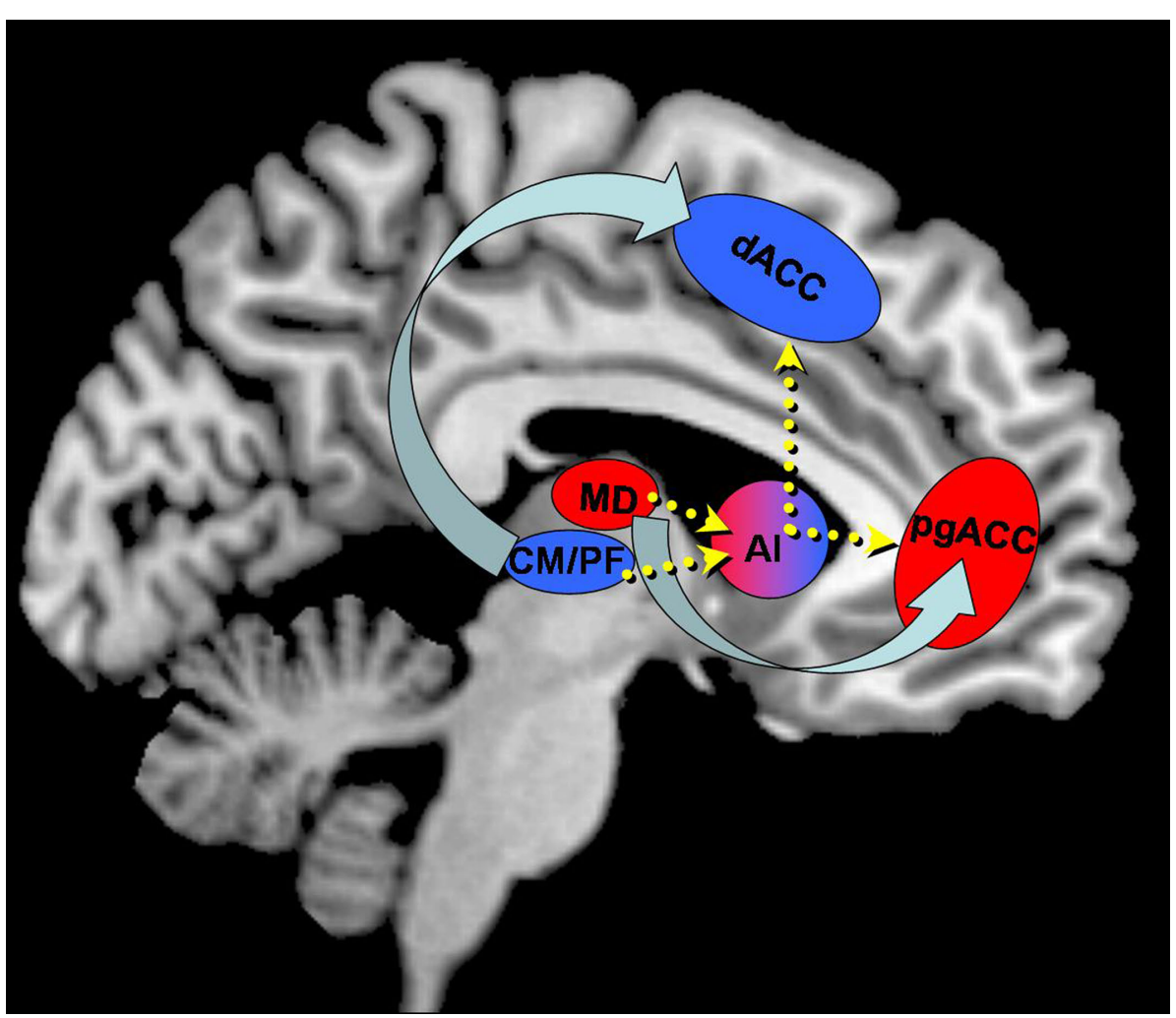

FIGURE 7 | Schematic representation of segregated and integrated processing of emotional and attentional dimensions during sexual stimulation. Distinct parcellations within thalamus reflect a rostro-caudal parcellation of ACC with direct connections of corresponding portions. The anterior insula (AI) may serve as an integrator with connections to both networks thus orchestrating functionally distinct processes. Arrow width does not indicate the strength of directionality.

The VA thalamic nucleus is one of the relay nuclei of the thalamus that have bidirectional connections both to basal ganglia and cortex (Haber and McFarland, 2001; McFarland and Haber, 2002; Haber and Calzavara, 2009). It is part of the oculomotor loop linking parts of the basal ganglia (caudate nucleus, globus pallidus, substantia nigra) and FEF (Alexander et al., 1986). Being associated with motor control and motor learning (Haber and McFarland, 2001), it holds cortical connections with the supplementary motor area, cingulate motor area, premotor cortex, PFC, and FEF.

In our experiment, we find main activation in VA and FEF during anticipation of sexual stimuli. This can be due to the motor preparatory component of the anticipation task, which is triggered by the sexual (motor relevant) content of the subsequent stimulus. This hypothesis is supported by our finding of coactivation of the precentral gyrus during the perception of erotic stimuli which has also been shown by previous studies investigating sexual perception (Mouras et al., 2003; Moulier et al., 2006).

\section{EXTRATHALAMIC ACTIVATIONS}

While hypothalamus and ventral striatum were not covered by our investigation, we could monitor sex effects in more dorsal parts of the ventral striatum:

\section{Caudate and putamen}

The caudate nucleus is part of several thalamocortical circuits as described by Alexander and colleagues (Alexander et al., 1986). It was shown to be involved in learning and memory (Oberg and Divac, 1975; Chozick, 1983; Packard and Knowlton, 2002). Moreover, an involvement in goal-directed behavior during erotic stimulation was suggested by Aron and colleagues (Aron et al., 2005). Studies investigating sexual processing found different activations in the caudate nucleus during erotic and emotional picture perception (Walter et al., 2008b). We found coactivation with both $\mathrm{MD}$ as well as $\mathrm{CM} / \mathrm{PF}$ in the medial head of caudate nucleus in both anticipation and picture period, which supports its involvement in separate thalamocortical circuits processing, among others, attention and emotion. As revealed by our results, sex during both anticipation and picture period elicited greater results in the right medial head of caudate nucleus, which underlines its involvement not only in sexual perception but also in sexual attention.

Connections from caudate nucleus have been described to $\mathrm{CM} / \mathrm{PF}$ in cats (Royce, 1978, 1983) and to MD in cats and monkeys (Showers, 1958; Macchi et al., 1984). We found a functional connectivity of the head of caudate nucleus with CM during anticipation period and with $\mathrm{MD}$ during picture perception. Our findings lend support to the previously described anatomical connections.

\section{Claustrum}

It has been suggested that the claustrum is involved in consciousness (Crick and Koch, 2005) and studies investigating sexual processing have been able to underline these findings (Mouras et al., 2003; Georgiadis et al., 2009, 2010). 
As revealed by previous studies, activation in the claustrum was often hard to differentiate from insular activity due to poor special resolution (Arnow et al., 2002). Making use of a high spatial resolution approach, we were able to distinguish between claustrum and insular activity. We found activation in the claustrum for erotic, but not for non-erotic picture perception. This finding supports the hypothesis of a previous study by Walter and colleagues that the claustrum is mainly involved in the processing of erotic stimuli (Walter et al., 2008b). As our stimuli were matched for emotional intensity, we even suggest the specificity of claustral activation to erotic stimuli, independent of their emotional content.

\section{LIMITATIONS}

While this is, to our knowledge, the first group study at ultra high field, enabling group inference on small anatomical structures, our group size was limited to a population of 10 male subjects. This group setting was chosen in a way to maximize homogeneity. Since some authors have proposed differences in sex-related activations in males and females (Hamann et al., 2004), the extrapolation of our results to a female population is thus questionable. However it was shown that specific activations were similar between males and females when different degrees of subjective ratings were controlled for (Karama et al., 2002) or when stimuli with equal sexual intensity were chosen (Walter et al., 2008a). We wanted to use an optimized paradigm of one single run and thus maximized sexual arousal of pictures for a male group, which also limited possible confounds of gender on the main effects. It remains subject to future studies to explore the newly described activation patterns in a female population. However, future studies may address a greater population not only to account for gender effects, but also to improve inference accuracy about a global population.

At the current stage, the subject selection criteria on ultra high fields are comparably strict which may change with the increase in safety experience on such systems and which will further allow the definition of an optimal sample size for group studies. While there are currently efforts to include increasing numbers of subjects into the same study on lower fields to tackle the same issue of generalizability (Fox and Lancaster, 2002), other scales may probably be more sensible given that larger smoothing in large scale population studies counteract the high spatial specificities for which our setup was chosen.

Despite the above mentioned advantages of high resolution $\mathrm{fMRI}$ at 7 Tesla, it suffers from reduced accuracy due to signal dropouts in ventral subcortical structures, such as parts of nucleus accumbens and hypothalamus, which are caused by magnetic field (B0) inhomogeneities at air-tissue boundaries. As these structures have been shown to be involved especially during sexual arousal (Mouras et al., 2003; Moulier et al., 2006; Walter et al., 2007), functional imaging sequences that reduce the signal loss in these structures are highly desirable. Higher resolution is possible and has been demonstrated (Speck et al., 2008). On the other hand, high temporal resolution needed for event-related studies is obtained at the cost of reduced volume coverage.

Other structures like the ventral pallidum show extremely short native T2* at 7 Tesla due to iron deposits (Hallgren and Sourander, 1958; Aoki et al., 1989) and thus very low signal intensity. The covered scan volume was based on strong prior hypotheses from previous similar studies of our own group (Heinzel et al., 2006; Walter et al., 2007, 2008a,b) and showed convincing effects within the regions of interest including thalamus, dACC and anterior insula. Due to this limited volume coverage, this study cannot answer specific question regarding sexual processing in distinct parts of the ventral and dorsal attention network or functional connectivity that would help to further defining small structures such as LHb.

It should also be noted that despite additional smoothing, we strongly emphasized our analysis on the detection of small structures such as thalamic subregions, while larger clusters of activation in bigger anatomical (e.g., cortical) regions could have benefited from greater smoothing kernels. Our approach was aimed at high spatial specificity necessary for the detection of small subcortical structures. As group statistics dependent on spatial smoothing to increase intersubject overlap we accepted that this may lead to reduced sensitivity in cortical areas.

For the performance of group statistics, all anatomical and functional data were transferred into Talairach space as offered by BrainVoyager QX. The anatomical alignment requires additional anatomical information which leads to an optimized overlap of subcortical regions and allows for a clear differentiation between distinct subcortical structures. In cortical areas, however, other means of non-linear normalization as provided by other software distributions may have been better suited.

\section{CONCLUSION}

Our results show the involvement of subcortical structures, especially the thalamus and caudate nucleus, in the core components of sexual processing and embed them into predescribed thalamocortical loops.

The emotional component of sexual stimuli is reflected in the coactivation of the mediodorsal thalamic nucleus together with the pregenual ACC that has been shown to encode the emotional component of sexual arousal on cortical level (Walter et al., 2008a).

In contrast, sexual attention is represented by activation in the centromedian/parafascicular and the lateral habenula complex. The $\mathrm{CM} / \mathrm{PF}$ that is connected to $\mathrm{dACC}$, showed a functional connectivity with the anterior insula during erotic anticipation, which we point to trigger the salience component of sexual attention.

The paraventricular thalamus, including the paraventricular $\mathrm{MD}, \mathrm{LD}$, and PT showed sex-specific activation, in accordance with connections to lateral hypothalamus, nucleus accumbens and cingulate cortex. This brings small parts of the thalamus back to a specific involvement in sexual processing which was so far not detectable using standard fMRI at lower fields.

For the first time, different components of sexual processing described in the literature and observed on cortical level could be related to distinct thalamocortical circuits involving the basal ganglia as a relay station of sexual arousal and attention.

\section{ACKNOWLEDGMENT}

This study was supported by the German Research Foundation (DFG-SFB 779, B. Bogerts and M. Walter) and a postgraduate research stipend to C. D. Metzger. We would like to thank Andreas Fügner and Gregor Szyzik for their skillfull assistance. 


\section{REFERENCES}

Alexander, G. E., DeLong, M. R., and Strick, P. L. (1986). Parallel organization of functionally segregated circuits linking basal ganglia and cortex. Annu. Rev. Neurosci. 9, 357-381.

Alexander, G. E., and Fuster, J. M. (1973). Effects of cooling prefrontal cortex on cell firing in the nucleus medialis dorsalis. Brain Res. 61, 93-105.

Allen, G. V., Saper, C. B., Hurley, K. M., and Cechetto, D. F. (1991). Organization of visceral and limbic connections in the insular cortex of the rat. J. Comp. Neurol. 311, 1-16.

Anand, A., Li, Y., Wang, Y., Wu, J., Gao, S., Bukhari, L., Mathews, V. P., Kalnin, A., and Lowe, M. J. (2005). Activity and connectivity of brain mood regulating circuit in depression: a functional magnetic resonance study. Biol. Psychiatry 57, 1079-1088.

Aoki, S., Okada, Y., Nishimura, K. Barkovich, A. J., Kjos, B. O., Brasch, R. C., and Norman, D. (1989). Normal deposition of brain iron in childhood and adolescence: MR imaging at $1.5 \mathrm{~T}$. Radiology 172, 381-385.

Arnow, B. A., Desmond, J. E., Banner, L. L., Glover, G. H., Solomon, A., Polan, M. L., Lue, T. F., and Atlas, S. W. (2002). Brain activation and sexual arousal in healthy, heterosexual males. Brain 125 , 1014-1023.

Aron, A., Fisher, H., Mashek, D. J., Strong, G., Li, H., and Brown, L. L. (2005). Reward, motivation, and emotion systems associated with early-stage intense romantic love. J. Neurophysiol. 94, 327-337.

Benjamini, Y., and Hochberg, Y. (1995). Controlling the false discovery rate: a practical and powerful approach to multiple testing. J. R. Stat. Soc. Ser. B 289-300.

Berendse, H. W., and Groenewegen, H. J. (1990). Organization of the thalamostriatal projections in the rat, with special emphasis on the ventral striatum. J. Comp. Neurol. 299, 187-228.

Berendse, H. W., and Groenewegen, H. J. (1991). Restricted cortical termination fields of the midline and intralaminar thalamic nuclei in the rat. Neuroscience 42, 73-102.

Brickenkamp, R., and Zillmer, E. (1998). d2 Test of Attention. Hogrefe: Goettingen.

Bush G., Luu P., and Posner M. I. (2000). Cognitive and emotional influences in anterior cingulate cortex. Trends Cogn. Sci. 4, 215-222.

Chozick, B. S. (1983). The behavioral effects of lesions of the corpus striatum: a review. Int. J. Neurosci. 19, 143-159.

Coolen, L. M., Peters, H. J., and Veening, J. G. (1997). Distribution of Fos immunoreactivity following mating versus anogenital investigation in the male rat brain. Neuroscience 77, 1151-1161.

Coolen, L. M., Veening, J. G., Petersen, D. W., and Shipley, M. T. (2003a). Parvocellular subparafascicular thalamic nucleus in the rat: anatomical and functional compartmentalization. J. Comp. Neurol. 463, 117-131.

Coolen, L. M., Veening, J. G., Wells, A. B., and Shipley, M. T. (2003b). Afferent connections of the parvocellular subparafascicular thalamic nucleus in the rat: evidence for functional subdivisions. J. Comp. Neurol. 463, 132-156.

Corbetta, M., and Shulman, G. L. (2002). Control of goal-directed and stimulusdriven attention in the brain. Nat. Rev. Neurosci. 3, 201-215.

Cornwall, J., and Phillipson, O. T. (1988a). Afferent projections to the dorsal thalamus of the rat as shown by retrograde lectin transport-I. The mediodorsal nucleus. Neuroscience 24, 1035-1049.

Cornwall, J., and Phillipson, O. T. (1988b). Afferent projections to the parafascicular thalamic nucleus of the rat, as shown by the retrograde transport of wheat germ agglutinin. Brain Res. Bull. 20, 139-150.

Crick, F. C., and Koch, C. (2005). What is the function of the claustrum? Philos. Trans. R. Soc. Lond. B. Biol. Sci. 360, 1271-1279.

Devinsky, O., Morrell, M. J., and Vogt, B. A. (1995). Contributions of anterior cingulate cortex to behaviour. Brain 118(Pt 1), 279-306.

Dosenbach, N. U. F., Fair, D. A., Cohen, A. L., Schlaggar, B. L., and Petersen, S. E. (2008). A dual-networks architecture of top-down control. Trends Cogn. Sci. 12, 99-105.

Drevets, W. C., Videen, T. O., Price, J. L., Preskorn, S. H., Carmichael, S. T., and Raichle, M. E. (1992). A functional anatomical study of unipolar depression. J. Neurosci. 12, 3628-3641.

Eckert, U., Kaufmann, J., Osoba, A., Steiner, J., Bogerts, B., and Walter, M. (2009). Cortical and subcortical connections of the thalamic nuclei CM and MD - a DTI tractography study. Neuroimage 47, 39-41.

Erickson, S. L., and Lewis, D. A. (2004). Cortical connections of the lateral mediodorsal thalamus in cynomolgus monkeys. J. Comp. Neurol. 473, 107-127.

Erickson, S. L., Melchitzky, D. S., and Lewis, D. A. (2004). Subcortical afferents to the lateral mediodorsal thalamus in cynomolgus monkeys. Neuroscience 129, 675-690.

Felton, T. M., Linton, L., Rosenblatt, J. S., and Morell, J. I. (1999). First and sec- ond order maternal behavior related afferents of the lateral habenula. Neuroreport 10, 883-887.

Fenelon, G., Francois, C., Percheron, G. and Yelnik, J. (1991). Topographic distribution of the neurons of the central complex (centre médianparafascicular complex) and of other thalamic neurons projecting to the striatum in macaques. Neuroscience 45, 495-510.

Fox, P. T., and Lancaster, Jack L. (2002). Opinion: mapping context and content: the BrainMap model. Nat. Rev. Neurosci. 3, 319-321.

François, C., Percheron, G., Parent, A. Sadikot, A. F., Fenelon, G., and Yelnik, J. (1991). Topography of the projection from the central complex of the thalamus to the sensorimotor striatal territory in monkeys. J. Comp. Neurol. 305, 17-34.

Friston, K. J. (1994). Functional and effective connectivity in neuroimaging: a synthesis. Hum. Brain Mapp. 2, 56-78.

Friston, K. J., Holmes, A. P., Worsley, K. J., Poline, J. -P; Frith, C. D., and Frackowiak, R. S. J. (1995). Statistical parametric maps in functional imaging: a general linear approach. Hum. Brain Mapp. 2, 189-210.

Genovese, C. R., Lazar, N. A., and Nichols, T. (2002). Thresholding of statistical maps in functional neuroimaging using the false discovery rate. Neuroimage 15, 870-878.

Georgiadis, J. R., Farrell, M. J., Boessen, R., Denton, D. A., Gavrilescu, M. and Kortekaas, R. (2010). Dynamic subcortical blood flow during male sexual activity with ecological validity: a perfusion fMRI study. Neuroimage 50, 208-216.

Georgiadis, J.R., Reinders,A.A. T.S.,Paans, A. M. J., Renken, R., and Kortekaas, R. (2009). Men versus women on sexual brain function: prominent differences during tactile genital stimulation, but not during orgasm. Hum. Brain Mapp. 30, 3089-3101.

Goebel, R., Esposito, F., and Formisano, E. (2006). Analysis of functional image analysis contest (FIAC) data with brainvoyager QX: From singlesubject to cortically aligned group general linear model analysis and self-organizing group independent component analysis. Hum. Brain Mapp. 27, 392-401.

Greicius, M. D., Flores, B. H., Menon, V. Glover, G. H., Solvason, H. B., Kenna, H., Reiss, A. L., and Schatzberg, A. F. (2007). Resting-state functional connectivity in major depression: abnormally increased contributions from subgenual cingulate cortex and thalamus. Biol. Psychiatry 62, 429-437.
Haber, S., and McFarland, N. R. (2001) The place of the thalamus in frontal cortical-basal ganglia circuits. Neuroscientist 7, 315-324.

Haber, S. N., and Calzavara, R. (2009). The cortico-basal ganglia integrative network: the role of the thalamus. Brain Res. Bull. 78, 69-74.

Hallgren, B., and Sourander, P. (1958). The effect of age on the non-haemin iron in the human brain. J. Neurochem. 3, 41-51.

Hamann, S., Herman, R. A., Nolan, C. L., and Wallen, K. (2004). Men and women differ in amygdala response to visual sexual stimuli. Nat. Neurosci. 7, 411-416.

Heeb, M. M., and Yahr, P. (1996). c-Fos immunoreactivity in the sexually dimorphic area of the hypothalamus and related brain regions of male gerbils after exposure to sex-related stimuli or performance of specific sexual behaviors. Neuroscience 72 , 1049-1071.

Heeb, M. M., and Yahr, P. (2001). Anatomical and functional connections among cell groups in the gerbil brain that are activated with ejaculation. J. Comp. Neurol. 439, 248-258.

Heinzel, A., Walter, M., Schneider, F., Rotte, M., Matthiae, C., Tempelmann, C., Heinze, H. J., Bogerts, B., and Northoff, G. (2006). Self-related processing in the sexual domain: a parametric event-related fMRI study reveals neural activity in ventral cortical midline structures. Soc. Neurosci. $1,41-51$.

Hikosaka, O. (2010). The habenula: from stress evasion to value-based decision-making. Nat. Rev. Neurosci. 11, 503-513.

Hikosaka, O., Sesack, S. R., Lecourtier, L., and Shepard, P. D. (2008). Habenula: crossroad between the basal ganglia and the limbic system. J. Neurosci. 28 , 11825-11829.

Isaacson, L. G., and Tanaka, D. (1986). Cholinergic and non-cholinergic projections from the canine pontomesencephalic tegmentum (Ch5 area) to the caudal intralaminar thalamic nuclei. Exp. Brain Res. 62, 179-188.

Johansen-Berg, H., Behrens, T. E. J., Sillery E., Ciccarelli O., Thompson, A. J., Smith, S. M., and Matthews, P. M. (2005). Functional-anatomical validation and individual variation of diffusion tractography-based segmentation of the human thalamus. Cereb. Cortex (New York, N.Y.: 1991), 15, 31-39.

Karama, S., Lecours, A. R., Leroux, J. M., Bourgouin, P., Beaudoin, G., Joubert, S., and Beauregard, M. (2002). Areas of brain activation in males and females during viewing of erotic film excerpts. Hum. Brain Mapp. 16, 1-13. 
Kinomura, S., Larsson, J., Gulyás, B., and Roland, P. E. (1996). Activation by attention of the human reticular formation and thalamic intralaminar nuclei. Science 271, 512-515.

Klein, J. C., Rushworth, M. F. S., Behrens, T. E. J., Mackay, C. E., Crespigny, A. J. de; D'Arceuil, H., and Johansen-Berg, H. (2010). Topography of connections between human prefrontal cortex and mediodorsal thalamus studied with diffusion tractography. NeuroImage 51, 555-564.

Klemm, W. R. (2004). Habenular and interpeduncularis nuclei: shared components in multiple-function networks. Med. Sci. Monit. 10, RA261-RA2673.

Krettek, J. E., and Price, J. L. (1977). The cortical projections of the mediodorsal nucleus and adjacent thalamic nuclei in the rat. J. Comp. Neurol. 171, 157-191.

Lang, P. J., Bradley, M. M., and Cuthbert, B. N. (2005). International Affective Picture System (IAPS): Affective Ratings of Pictures and Instruction Manual. Herausgegeben von Gainesville Technical Report A-6. University of Florida.

Litt, A., Plassmann, H., Shiv, B., and Rangel, A. (2010). Dissociating valuation and saliency signals during decision-making. Cereb. Cortex (in press). http://cercor.oxfordjournals. org/content/early/2010/05/05/cercor. bhq065.full.pdf+html

Macchi, G., Bentivoglio, M., Molinari, M., and Minciacchi, D. (1984). The thalamo-caudate versus thalamocortical projections as studied in the cat with fluorescent retrograde double labeling. Exp. Brain Res. 54, 225-239.

Mai, J., Assheuer, J., and Paxinos, G. (2004). Atlas of the Human Brain. san Diego: Academic Press.

Matsumoto, M., and Hikosaka, O. (2007). Lateral habenula as a source of negative reward signals in dopamine neurons. Nature 447, 1111-1115.

Matsumoto, M., and Hikosaka, O. (2009). Representation of negative motivational value in the primate lateral habenula. Nat. Neurosci. 12, 77-84.

Matsumoto, N., Minamimoto, T., Graybiel, A. M., and Kimura, M. (2001). Neurons in the thalamic CM-Pf complex supply striatal neurons with information about behaviorally significant sensory events. J. Neurophysiol. 85, 960-976.

McFarland, N. R., and Haber, S. N. (2002). Thalamic relay nuclei of the basal ganglia form both reciprocal and nonreciprocal cortical connections, linking multiple frontal cortical areas. J. Neurosci. 22, 8117-8132.
Moruzzi, G., and Magoun, H. W. (1949). Brain stem reticular formation and activation of the EEG. Electroencephalogr. Clin. Neurophysiol. 1, 455-473.

Moulier, V., Mouras, H., Pélégrini-Issac, M., Glutron, D., Rouxel, R., Grandjean, B., Bittoun, J., and Stoléru, S. (2006). Neuroanatomical correlates of penile erection evoked by photographic stimuli in human males. Neuroimage 33, 689-699.

Mouras, H., Stoléru, S., Bittoun, J., Glutron, D., Pélégrini-Issac, M., Paradis, A., and Burnod, Y. (2003). Brain processing of visual sexual stimuli in healthy men: a functional magnetic resonance imaging study. Neuroimage 20, 855-869.

Mufson, E. J., and Mesulam, M. M. (1984). Thalamic connections of the insula in the rhesus monkey and comments on the paralimbic connectivity of the medial pulvinar nucleus. J. Comp. Neurol. 227, 109-120.

Nakano, K., Hasegawa, Y., Tokushige, A., Nakagawa, S., Kayahara, T., and Mizuno, N. (1990). Topographical projections from the thalamus, subthalamic nucleus and pedunculopontine tegmental nucleus to the striatum in the Japanese monkey, Macaca fuscata. Brain Res. 537, 54-68.

Nichols, T., Brett, M., Andersson, J., Wager, T., and Poline, J. (2005). Valid conjunction inference with the minimum statistic. Neuroimage 25, 653-660.

Oberg, R. G., and Divac, I. (1975). Dissociative effects of selective lesions in the caudate nucleus of cats and rats. Acta Neurobiol. Exp. 35, 647-659.

Oyoshi, T., Nishijo, H., Asakura, T., Takamura, Y., and Ono, T. (1996). Emotional and behavioral correlates of mediodorsal thalamic neurons during associative learning in rats. $J$. Neurosci. 16, 5812-5829.

Packard, M. G., and Knowlton, B. J. (2002). Learning and memory functions of the basal ganglia. Annu. Rev. Neurosci. 25, 563-593.

Powell, T. P., and Cowan, W. M. (1954). The connexions of the midline and intralaminar nuclei of the thalamus of the rat. J. Anat. 88, 307-319.

Price, J. L. (1999). Prefrontal cortical networks related to visceral function and mood. Ann. N.Y. Acad. Sci. 877, 383-396.

Price, J. L., Carmichael, S. T., and Drevets, W. C. (1996). Networks related to the orbital and medial prefrontal cortex; a substrate for emotional behavior? Prog. Brain Res. 107, 523-536.

Ray, J. P., and Price, J. L. (1992). The organization of the thalamocorti- cal connections of the mediodorsal thalamic nucleus in the rat, related to the ventral forebrain-prefrontal cortex topography. J. Comp. Neurol. 323, 167-197.

Ray, J. P., and Price, J. L. (1993). The organization of projections from the mediodorsal nucleus of the thalamus to orbital and medial prefrontal cortex in macaque monkeys. J. Comp. Neurol. 337, 1-31.

Redouté, J., Stoléru, S., Grégoire, M. C., Costes, N., Cinotti, L., Lavenne, F., Le Bars, D., Forest, M. G., and Pujol, J. F. (2000). Brain processing of visual sexual stimuli in human males. Hum. Brain Mapp. 11, 162-177.

Reep, R. L., and Winans, S. S. (1982a) Afferent connections of dorsal and ventral agranular insular cortex in the hamster Mesocricetus auratus. Neuroscience 7, 1265-1288.

Reep, R. L., and Winans, S. S. (1982b). Efferent connections of dorsal and ventral agranular insular cortex in the hamster, Mesocricetus auratus. Neuroscience 7, 2609-2635.

Rorden, C., Karnath, H. O., and Bonilha, L. (2007). Improving lesion-symptom mapping. J. Cogn. Neurosci. 19, 1081-1088.

Rose, J. E., and Woolsey, C. N. (1949). Organization of the mammalian thalamus and its relationships to the cerebral cortex. Electroencephalogr. Clin. Neurophysiol. 1, 391-403; discussion 403-404.

Royce, G. J. (1978). Autoradiographic evidence for a discontinuous projection to the caudate nucleus from the centromedian nucleus in the cat. Brain Res. 146, 145-150.

Royce, G. J. (1983). Cortical neurons with collateral projections to both the caudate nucleus and the centromedianparafascicular thalamic complex: a fluorescent retrograde double labeling study in the cat. Exp. Brain Res. 50, 157-165.

Royce, G. J., and Mourey, R. J. (1985). Efferent connections of the centromedian and parafascicular thalamic nuclei: an autoradiographic investigation in the cat. J. Comp. Neurol. 235, 277-300.

Ryszka, A., and Heger, M. (1979). Afferent connections of the laterodorsal thalamic nucleus in the rat. Neurosci. Lett. 15, 61-64

Sadikot, A. F., Parent, A., and François, C. (1992). Efferent connections of the centromedian and parafascicular thalamic nuclei in the squirrel monkey: a PHA-L study of subcortical projections. J. Comp. Neurol. 315 , 137-159.

Salas, R., Baldwin, P., and Biasi, M de Montague, P. R. (2010). BOLD responses to negative reward predic- tion errors in human habenula. Front. Hum. Neurosci. 4:36. doi: 10.3389/ fnhum.2010.00036.

Sartorius, A., and Henn, F. A. (2007). Deep brain stimulation of the lateral habenula in treatment resistant major depression. Med. Hypotheses 69, 1305-1308.

Sartorius, A., Kiening, K. L., Kirsch, P., Gall, C. C. von; Haberkorn, U., Unterberg, A. W., Henn, Fritz A., and Meyer-Lindenberg, A. (2010). Remission of major depression under deep brain stimulation of the lateral habenula in a therapy-refractory patient. Biol. Psychiatry 67, e9-e11.

Sartorius, A., and Meyer-Lindenberg, A. (2009). Deep brain stimulation of the lateral habenula to treat depression. Front. Neurosci. 3:272.

Seeley, W. W., Menon, V., Schatzberg, A. F., Keller, J., Glover, G. H., Kenna, H., Reiss, A. L., and Greicius, M. D. (2007). Dissociable intrinsic connectivity networks for salience processing and executive control. J. Neurosci. 27, 2349-2356.

Shibata, H. (2000). Organization of retrosplenial cortical projections to the laterodorsal thalamic nucleus in the rat. Neurosci. Res. 38, 303-311.

Showers, M. J. (1958). Correlation of medial thalamic nuclear activity with cortical and subcortical neuronal arcs. J. Comp. Neurol. 109, 261-315.

Speck, O., Stadler, J., and Zaitsev, M. (2008). High resolution single-shot EPI at 7T. MAGMA 21, 73-86.

Temel, Y., Visser-Vandewalle, V., Ackermans, L., and Beuls, E. A. M. (2004). Thalamus and penile erection. Int. J. Impot. Res. 16, 505-511.

Thompson, S. M., and Robertson, R. T. (1987). Organization of subcortical pathways for sensory projections to the limbic cortex. II. Afferent projections to the thalamic lateral dorsal nucleus in the rat. J. Comp. Neurol. 265, 189-202.

van der Werf, Y. D., Witter, M. P., and Groenewegen, H. J. (2002). The intralaminar and midline nuclei of the thalamus. Anatomical and functional evidence for participation in processes of arousal and awareness. Brain Res. Brain Res. Rev. 39, 107-140.

van Groen, T., and Wyss, J. M. (1992). Projections from the laterodorsal nucleus of the thalamus to the limbic and visual cortices in the rat. J. Comp. Neurol. 324, 427-448.

Veening, J. G., and Coolen, L. M. (1998). Neural activation following sexual behavior in the male and female rat brain. Behav. Brain Res. 92, 181-193.

Vertes, R. P. (2006). Interactions among the medial prefrontal cortex, hippocampus and midline thalamus in 
emotional and cognitive processing in the rat. Neuroscience 142, 1-20.

Vogt, B. A., Pandya, D. N., and Rosene, D. L. (1987a). Cingulate cortex of the rhesus monkey: I. Cytoarchitecture and thalamic afferents. J. Comp. Neurol. 262, 256-270.

Vogt, B. A., Pandya, D. N., and Rosene, D. L. (1987b). Cingulate cortex of the rhesus monkey: I. Cytoarchitecture and thalamic afferents. J. Comp. Neurol. 262, 256-270.

Walter, M., Bermpohl, F., Mouras, H., Schiltz, K., Tempelmann, C., Rotte, M. Heinze H. J., Bogerts B., and Northoff G. (2008a). Distinguishing specific sexual and general emotional effects in fMRI-subcortical and cortical arousal during erotic picture viewing. Neuroimage 40, 1482-1494.
Walter, M., Henning, A., Grimm, S., Schulte, R. F., Beck, J., Dydak, U., Dydak U., Schnepf B., Boeker H., Boesiger P., and Northoff G. (2009). The relationship between aberrant neuronal activation in the pregenual anterior cingulate, altered glutamatergic metabolism, and anhedonia in major depression. Arch. Gen. Psychiatry 66, 478-486.

Walter, M., Stadler, J., Tempelmann, C., Speck, O., and Northoff, G. (2008b). High resolution fMRI of subcortical regions during visual erotic stimulation at 7 T. MAGMA 21, 103-111.

Walter, M., Witzel, J., Wiebking, C., Gubka, U., Rotte, M., Schiltz, K., Bermpohl F., Tempelmann C., Bogerts B., Heinze H. J., and Northoff G. (2007). Pedophilia is linked to reduced activation in hypothalamus and lateral prefrontal cortex during visual erotic stimulation. Biol. Psychiatry 62, 698-701.

Zaitsev, M., Hennig, J., and Speck, O. (2004). Point spread function mapping with parallel imaging techniques and high acceleration factors: fast, robust, and flexible method for echo-planar imaging distortion correction. Magn. Reson. Med. 52, 1156-1166.

Zhang, D., Snyder, A. Z., Shimony, J. S., Fox, M. D., and Raichle, M. E. (2010). Noninvasive functional and structural connectivity mapping of the human thalamocortical system. Cereb. Cortex (New York, N.Y.: 1991), 20, 1187-1194.

Conflict of Interest Statement: The authors declare that the research was conducted in the absence of any commercial or financial relationships that could be construed as a potential conflict of interest.
Received:30July2010; accepted:20September 2010; published online: 01 November 2010. Citation: Metzger CD, Eckert U, Steiner J, Sartorius A, Buchmann JE, Stadler J, Tempelmann C, Speck O, Bogerts B, Abler $B$ and Walter M (2010) High field fMRI reveals thalamocortical integration of segregated cognitive and emotional processing in mediodorsal and intralaminar thalamic nuclei. Front. Neuroanat. 4:138. doi: 10.3389/fnana.2010.00138

Copyright (C) 2010 Metzger, Eckert, Steiner, Sartorius, Buchmann, Stadler, Tempelmann, Speck, Bogerts, Abler and Walter. This is an open-access article subject to an exclusive license agreement between the authors and the Frontiers Research Foundation, which permits unrestricted use, distribution, and reproduction in any medium, provided the original authors and source are credited. 